\title{
Structure and Dynamics of Confined Liquids: Challenges and Perspectives for the X-ray Surface Forces Apparatus
}

Henning Weiss, ${ }^{\dagger}$ Hsiu-Wei Cheng, ${ }^{\ddagger}$ Julian Mars, ${ }^{\dagger, \S}$ Hailong Li, ${ }^{\dagger}$ Claudia Merola, ${ }^{\ddagger}$ Frank Uwe Renner, ${ }^{\| \oplus}$ Veijo Honkimäki, ${ }^{\perp}$ Markus Valtiner, ${ }^{*},+, \#$ and Markus Mezger ${ }^{*}, \dagger, \S \subset$

${ }^{\dagger}$ Max Planck Institute for Polymer Research, Ackermannweg 10, 55128 Mainz, Germany

${ }^{\ddagger}$ Institute of Applied Physics, Vienna Institute of Technology, Wiedner Hauptstrasse 8-10/E134, 1040 Wien, Austria

${ }^{\S}$ Institute of Physics, Johannes Gutenberg University Mainz, 55128 Mainz, Germany

"Institute for Materials Research, Hasselt University, 3590 Diepenbeek, Belgium

${ }^{\perp}$ ESRF-European Synchrotron Radiation Facility, Avenue des Martyrs 71, 38043 Grenoble, Cedex 9, France

${ }^{\#}$ Max-Planck-Institut für Eisenforschung GmbH, Max-Planck-Strasse 1, 40237 Düsseldorf, Germany

\section{Supporting Information}

ABSTRACT: The molecular-scale structure and dynamics of confined liquids has increasingly gained relevance for applications in nanotechnology. Thus, a detailed knowledge of the structure of confined liquids on molecular length scales is of great interest for fundamental and applied sciences. To study confined structures under dynamic conditions, we constructed an in situ X-ray surface forces apparatus (X-SFA). This novel device can create a precisely controlled slit-pore confinement down to dimensions on the $10 \mathrm{~nm}$ scale by using a cylinder-on-flat geometry for the first time. Complementary structural information can be obtained by simultaneous force measurements and X-ray scattering experiments. The in-plane structure of liquids parallel to
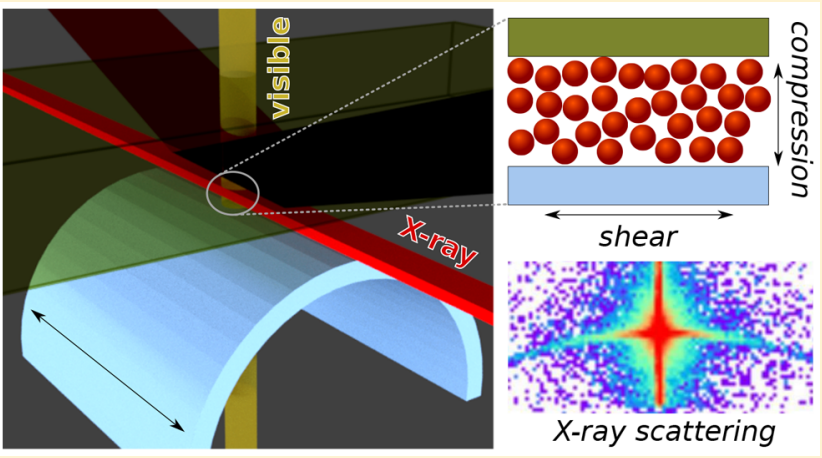
the slit pore and density profiles perpendicular to the confining interfaces are studied by X-ray scattering and reflectivity. The normal load between the opposing interfaces can be modulated to study the structural dynamics of confined liquids. The confinement gap distance is tracked simultaneously with nanometer precision by analyzing optical interference fringes of equal chromatic order. Relaxation processes can be studied by driving the system out of equilibrium by shear stress or compression/decompression cycles of the slit pore. The capability of the new device is demonstrated on the liquid crystal 4'-octyl-4-cyano-biphenyl (8CB) in its smectic A (SmA) mesophase. Its molecular-scale structure and orientation confined in $100 \mathrm{~nm}$ to $1.7 \mu \mathrm{m}$ slit pores was studied under static and dynamic nonequilibrium conditions.

\section{INTRODUCTION}

Confined liquids play an important role in many technical applications and processes. For example, the dynamics of electrolytes in nanoscale confinement are relevant to the development of novel electric double-layer capacitors and electrochemical processes. ${ }^{1-4}$ In heterogeneous catalysis, the performance of a reaction has been shown to be sensitive to the molecular-scale liquid structure in a nanoporous material. ${ }^{5}$ In particular for complex electrolytes with heterogeneities on the nanometer length scale, ${ }^{6-9}$ confinement can qualitatively alter structural properties. High-speed printing and film deposition on nanoscopically rough surfaces depend on the wetting property of surfactants in small pores. Thus, a detailed knowledge of the structure of confined liquids on molecular length scales is of great interest for fundamental and applied sciences. ${ }^{10}$

When confinement approaches the molecular dimensions of liquids, structural and dynamical properties can differ significantly from their bulk behavior $^{11-13}$ (Figure $1 \mathrm{a}-\mathrm{d}$ ). It has been shown that, depending on the intermolecular forces, soft matter adjacent to a solid and near surfaces can exhibit interfacial regions of reduced density, ${ }^{14-16}$ interfacial freezing, ${ }^{17-19}$ interfacial melting, ${ }^{20-23}$ molecular layering, ${ }^{24-31}$ molecular orientation, ${ }^{32}$ or specific lateral molecular arrangements. ${ }^{33}$ Furthermore, the phase diagram of confined liquids can differ significantly from the bulk. ${ }^{34,35}$ Such interface-induced structures can strongly affect the dynamics in a confined system. Prominent examples include increasing friction under lateral shear ${ }^{36,37}$ as well as increased reactivity at electrified interfaces ${ }^{3}$ (Figure 1e,f).

Today, most structural information on the molecular-scale arrangement of confined fluids is obtained by computer simulations ${ }^{40,41}$ or indirectly deduced from force-distance curves measured using a surface forces apparatus (SFA, Figure 2d), colloidal probe ${ }^{42-44}$ (Figure 2e), or atomic force microscope (AFM). In particular, the SFA is an established experimental

Received: May 2, 2019

Revised: October 15, 2019

Published: October 15, 2019 
(a)

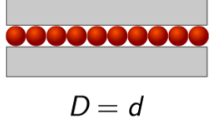

(b)

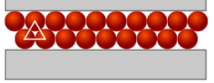

$D=(1+\sqrt{3} / 2) d$ (c)

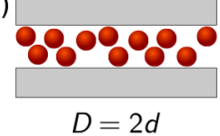

(d)

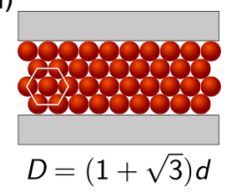

(e)

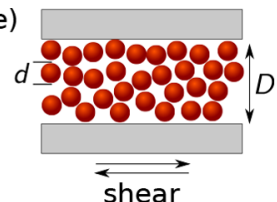

(f)

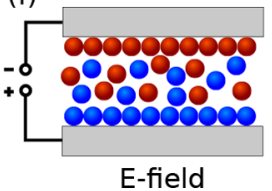

Figure 1. Structure of hard spheres with diameter $d$ in a 2D slit-pore confinement at gap widths of $D=d(\mathrm{a}), D=\left(1+\frac{\sqrt{3}}{2}\right) d(\mathrm{~b}), D=2 d$ (c), and $D=(1+\sqrt{3}) d(\mathrm{~d})$. In 3D, adjacent layers of close-packed spheres ( $b$ and $d$ ) arrange with respect to their tetrahedral gaps. Dynamic response of the system after applying shear stress (e) or an electric potential (f). (a)

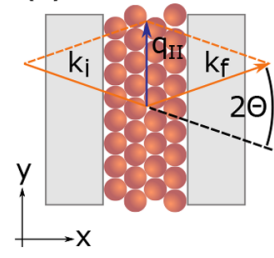

(d)

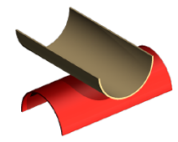

(e) (b)

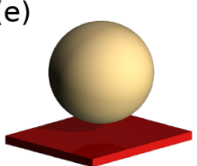

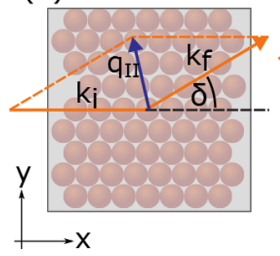

(f)

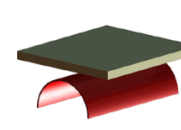

(c)

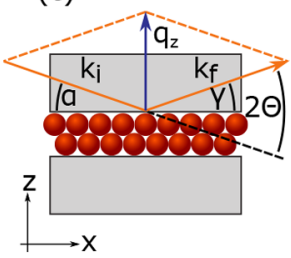

(g)
Figure 2. Overview of X-SFA geometries to study confined liquids by $\mathrm{X}$-ray scattering. Scattering angles follow the six-circle notation by Vlieg et al. ${ }^{38}$ with incident angle $\alpha$, exit angle $\gamma$, in-plane angle $\delta$, and total scattering angle $2 \theta$. (a) In transmission geometry, incident and scattered X-ray beams (orange) penetrate both confining slit-pore walls (gray). ${ }^{39}$ (b) In in-plane scattering geometry, the incident beam $\alpha=0$ is aligned parallel to the confining surfaces. At $\gamma=0$, the momentum transfer of the scattering vector $q=k_{\mathrm{f}}-k_{\mathrm{i}}$ points in the direction perpendicular to the surface normal (i.e., $q_{\|}$in the $x-y$ plane). (c) Specular reflectivity (XRR) with $\alpha=\gamma$ and $q=q_{z}$ parallel to the surface normal of the slit-pore walls. This configuration probes interfacial profiles along the $z$ direction (i.e., across the slit pore). Confinement can be created in the standard SFA crossed-cylinders geometry (d), the colloidal probe sphere-on-flat geometry (e), the cylinder-on-flat geometry (f), or the plane-plane geometry (g).

setup to simultaneously measure forces and distances across two surfaces approaching each other on a range from micrometers down to nanometers with nanometer precision.

This article is structured as follows. First, we give an overview of past and present experimental approaches to address the molecular-scale structures and dynamics of confined fluids by elastic scattering and force measurements. We then describe a novel design for an X-ray surface forces apparatus (X-SFA) in cylinder-on-flat geometry combining the capabilities of an SFA setup with X-ray scattering techniques. Our first X-SFA experiments on smectic liquid crystal $4^{\prime}$-octyl-4-cyano-biphenyl $(8 \mathrm{CB})$ in confinement are presented. They demonstrate the feasibility of the cylinder-on-flat geometry and the increased sensitivity and capability of the new instrument. The article closes with an outlook in which we discuss the potential of the new setup to answer current scientific questions in the field of soft condensed matter, develop ideas for future instruments, and outline first concepts for experiments that will become possible with new synchrotron sources. 45,46

\section{EXPERIMENTS PROBING THE STRUCTURE AND DYNAMICS OF CONFINED LIQUIDS}

An SFA is used to study liquids under controlled slit-pore confinement with gap dimensions down to the molecular length scale. ${ }^{47}$ Furthermore, a SFA can very precisely apply stress to the confined material and measure its viscoelastic response. From SFA experiments, limited structural information can be obtained from refractive index measurements. ${ }^{48,49}$ However, force measurements in an SFA, colloidal probe, or AFM, actually do not directly probe the spatial arrangement of the molecules. ${ }^{50,51}$ Instead, structural information is inferred from force measurements using model assumptions to interpret the force data. ${ }^{52,53}$ On the other hand, X-ray scattering and reflectivity (XRR) are powerful techniques to directly probe structures on molecular length scales in the bulk and at interfaces. ${ }^{54-56}$ Today, highbrilliant synchrotron sources allow us to study the structural dynamics at interfaces and in small sample volumes in situ on submicrosecond time scales. ${ }^{57-60}$

For instance, extensive work has been conducted on the molecular-scale structure of soft matter confinement in porous bulklike materials using X-ray and neutron scattering techniques. ${ }^{35,61-65}$ Today, nearly monodisperse cylinders in silica or aluminum oxide (AAO) with diameters ranging from $5 \mathrm{~nm}$ to $1 \mu \mathrm{m}$ are available. ${ }^{66-70}$ The macroscopic amount of liquid confined inside millions of uniform pores allows the use of bulk techniques such as dielectric spectroscopy, ${ }^{71,72} \mathrm{NMR},{ }^{73}$ optical birefringent, ${ }^{35}$ and QENS. ${ }^{74}$ However, even for such well-defined pores, in scattering experiments some of the structural information on the confined liquids is lost because of the intrinsic average in cylinder symmetry. In disordered and polydisperse porous materials, a quantitative interpretation of scattering data is even more challenging. Because of the Gibbs-Thomson effect, ${ }^{75,76}$ the large curvature in nanosized porous materials strongly affects the phase behavior of confined fluids. ${ }^{77}$ Moreover, dynamic studies during shear, compression, and decompression are intrinsically difficult to realize experimentally in a porous system. Therefore, to probe the intrinsic structure and dynamics of liquids confined between two parallel walls, other geometries have to be employed.

An X-ray surface forces apparatus (X-SFA) simultaneously combines the two complementary experimental techniques of force-distance measurements and X-ray scattering. ${ }^{47}$ This unique combination promises results not accessible by any other experimental method. Moreover, an SFA allows moving the pore walls vertically and laterally with respect to each other. Therefore, the development of an X-SFA that allows driving the structure of confined liquids out of equilibrium by lateral and vertical motion of the pore walls while recording its relaxation by scattering techniques will open up new ways to study the structural relaxation dynamics of confined liquids under shear and load.

The first X-SFA for transmission experiments (Figure 2a) in crossed-cylinder geometry (Figure 2d) was introduced in 1993 by Israelachvili's and Safinya's groups. ${ }^{36,78-84}$ Experiments on liquid crystal 4'-octyl-4-cyano-biphenyl (8CB) demonstrated that a 10 -nm-thick $8 \mathrm{CB}$ film provides sufficient scattering intensity to extract structural information on the molecular orientation induced by the confinement between the two mica cylinders.

Later, two different teams, lead by Reichert and Mugele at ID10C, ${ }^{85}$ ESRF and van der Veen and Heuberger at the Swiss 
Light Source, ${ }^{86}$ used a similar geometry. The latter team also explored the potential of an X-SFA for X-ray reflectivity (Figure 2c) using the crossed-cylinder geometry (Figure 2d). ${ }^{87-90}$ However, because of the large curvature of the cylinders, data can be taken only at large vertical momentum transfers $q_{z}>4.0 \mathrm{~nm}^{-1}$. This precludes conventional XRR measurements in the Fresnel regime. Therefore, the structure of the confined liquid can be deduced only from crystal truncation rods (CTR) using a rather complex data analysis. ${ }^{90}$ No structural in-plane information (Figure 2b) is accessible in this geometry because a defined slitpore confinement is established only at the very center of the crossed cylinders.

The group of Seeck at PETRA III designed two different $\mathrm{X}$-SFAs in plane-plane geometry (Figure $2 \mathrm{~g}$ ). ${ }^{91,92}$ In their current version, confining walls are made of two diamond single crystals commonly used for diamond anvil cells. With a maximum possible force of $100 \mathrm{~N}$ applied to the $200-\mu \mathrm{m}$-diameter diamond culets in the crystallographic (100) direction, pressures of up to $3 \mathrm{GPa}$ can be reached to obtain molecular-scale confinement. Parallel alignment of the opposing macroscopic planar interfaces can be rather challenging. Furthermore, in some cases the information extracted from the scattering patterns is limited by the diamonds' surface roughness of approximately $1 \mathrm{~nm} \mathrm{rms}$ (root mean square) and up to $1.4 \mathrm{~nm}$ peak-to-valley. In X-ray scattering and specular reflectivity experiments on confined liquid benzene, a gap thickness down to $5 \mathrm{~nm}$ was reached. ${ }^{92}$ In an experiment on carbon tetrachloride, crystal-liquid coexistence was found in slit-pore confinement ${ }^{93}$

Recently, a device enabling shear measurements while performing X-ray experiments was realized by Kurihara's group. ${ }^{94}$ Using a crossed-cylinder geometry, scattering experiments probe the molecular structure parallel to the two confining surfaces.

For neutron scattering experiments, devices usually feature large confined areas to accommodate large beam footprints. Sizes up to square centimeters might aggravate a reproducible and precise confinement over the entire area. Particles trapped in confinement are prone to corrupt measurements. The larger the confined areas, the more challenging the elimination of contaminants.

Kuhl's group succeeded in creating a $100 \mathrm{~nm}$ slit-pore confinement with $\pm 15 \mathrm{~nm}$ local deviations. ${ }^{95,96}$ Their cell allows shear experiments in the frequency range between 0.001 and $20 \mathrm{~Hz}$. This setup was primarily used to investigate confined polymer films. ${ }^{97}$

Another instrument used to study the structure of confined soft matter films upon applying a controlled external pressure using neutron reflectometry was developed by de Vos et al. ${ }^{98}$ Here, confinement down to the nanometer level and over large areas is achieved by an inflatable flexible membrane approach. With this approach, they probed the effects of confinement on a poly(vinyl pyrrolidone) gel layer in water, a polyelectrolyte multilayer in water, and the lamellar structures of a $\mathrm{D}_{2} \mathrm{O}$ swollen stack of supported lipid bilayers. Over the last few years, it has been demonstrated that this setup can provide detailed insight into the equilibrium structure of confined soft matter at known confining pressures. Various studies on polyelectrolyte brushes and lipid bilayers ${ }^{99-102}$ have been reported. However, the instrument does not allow for lateral motion of the confining surfaces against each other.

Changes in the interfacial profiles of hexadecane under shear rates of up to $1000 \mathrm{~Hz}$ have been studied by neutron reflectivity in a cone-plate rheometer. ${ }^{103-105}$ As an alternative setup at P10, PETRA III using a modified plate-plate rheometer was employed to study the influence of shear forces on the structure of bulk liquids by X-ray scattering techniques. ${ }^{106,107}$ However, because of their large sample thicknesses most instruments based on conventional rheometers are not able to reach high enough shear rates to detect fast interfacial relaxation processes on the molecular length scale such as adsorption and desorption. ${ }^{108,109}$

In this article, we present a novel design for an X-SFA in cylinder-on-flat geometry (Figure 2f) for in-plane scattering (Figure 2b) and specular XRR (Figure 2c). Data, obtained in these complementary scattering geometries, caries information on the normal and lateral molecular arrangement of soft matter in slit-pore confinement. Furthermore, compared to the crossed cylinder geometry (Figure 2d), the cylinder-on-flat geometry provides significantly more confined sample volume for scattering experiments. Using white light interferometry, this device can realize a controlled confinement in slit-pore geometry with gap thicknesses $D$ ranging from several $10 \mu \mathrm{m}$ down to the $10 \mathrm{~nm}$ length scale. The instrument is able to apply lateral (shear stress) and vertical (compression/decompression) relative motion of the confining solids and monitor structural changes in situ. Simultaneously, normal and frictional forces can be measured by strain gauges. Thus, X-SFA experiments can provide complementary information to force-distance measurements and conventional X-ray and neutron scattering studies in nanoporous materials. In particular, our new X-SFA can be used to study relaxation processes by driving the system out of equilibrium by shear stress or compression and decompression.

\section{X-RAY SURFACE FORCES APPARATUS}

General Design. In our new X-SFA setup (Figures 3 and 4), confinement is realized between a stationary upper planar

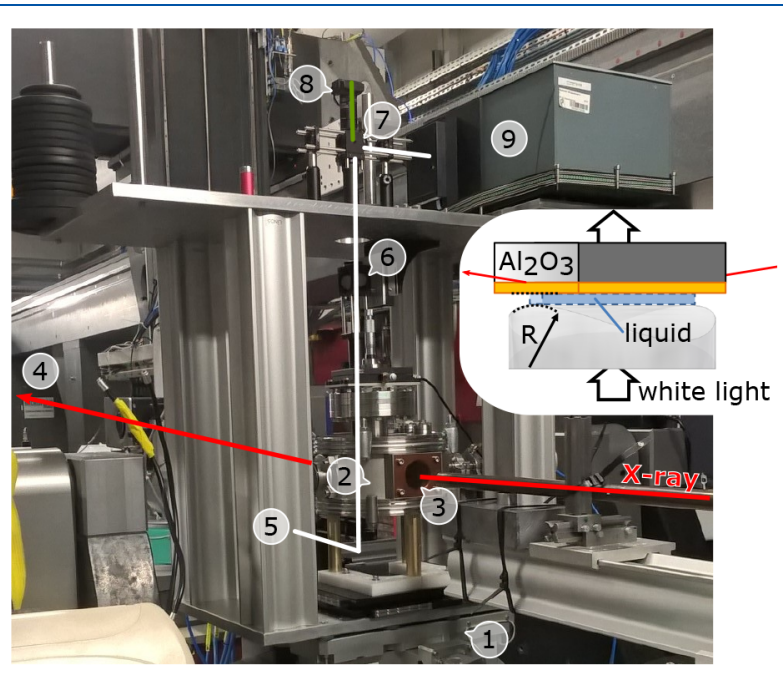

Figure 3. Photography of the X-SFA setup mounted on the HEMD diffractometer (1) at ID31 ESRF. Lines indicate optical (white and green light) and X-ray (red) beam paths. The SFA with the confined liquid (inset) is located at the diffractometer's rotation center inside a helium-filled stainless steel chamber (2). X-rays enter and leave the chamber nearly horizontally through $80 \mu \mathrm{m}$ Kapton windows (3) and are recorded on $2 \mathrm{D}$ detectors (4). White light from a fiber light source (5) is fed in via a mirror from below. The microscope objective (6), beam splitter (7), top-view CCD camera (8), and spectrometer (9) are mounted above the sample chamber on the upper instrument level.

surface and a cylindrically curved lower surface in a flat-oncylinder geometry (Figure 2f). Both surfaces in direct contact with the liquid are atomically smooth with a roughnesses below 

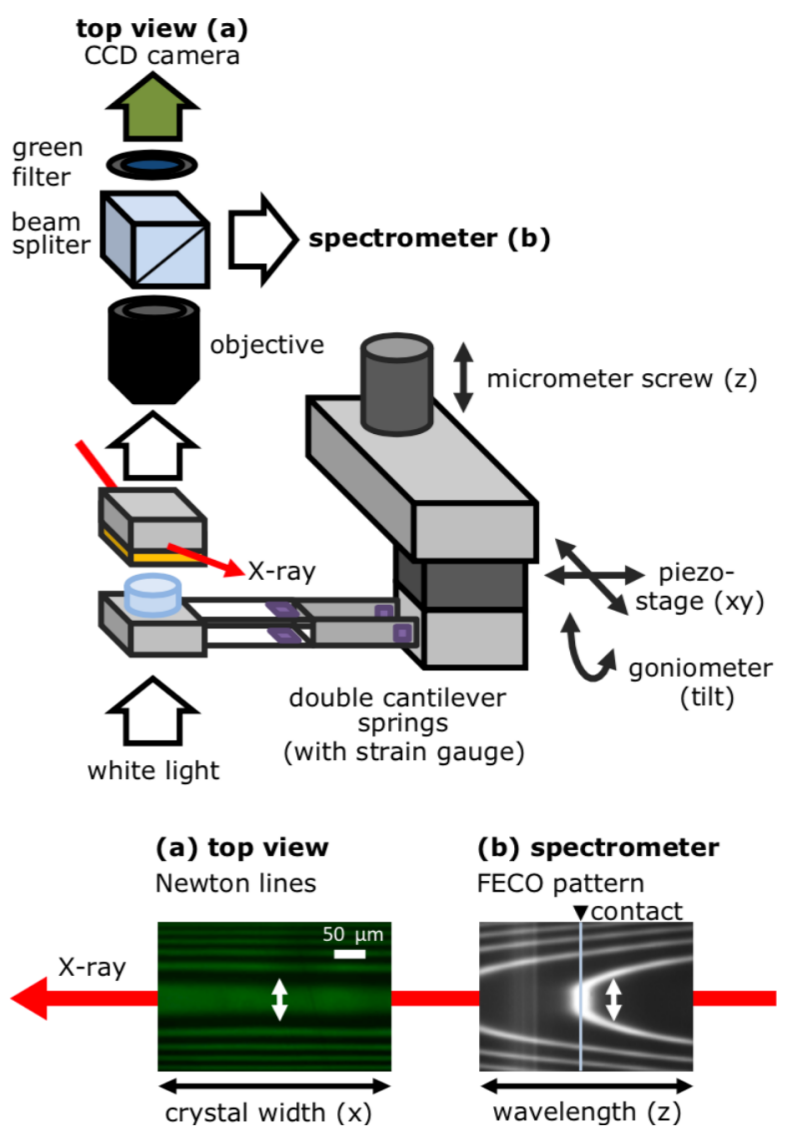

Figure 4. Schematic of the X-SFA setup including double-cantilever springs with strain gauges and optics for recording Newton's interference and fringes of equal chromatic order (FECO). (a) Top view of the confined $8 \mathrm{CB}$ by video microscopy indicating perfect parallel alignment of the opposing surfaces. (b) FECOs are used to determine the gap width.

$0.5 \mathrm{~nm} \mathrm{rms} \mathrm{(Figure} \mathrm{S2).} \mathrm{Their} \mathrm{surface} \mathrm{properties} \mathrm{can} \mathrm{be}$ tuned by functionalization with self-assembled monolayers (SAMs).

As a stationary upper surface, we use an atomically smooth template stripped gold layer on a corundum $\left(\mathrm{Al}_{2} \mathrm{O}_{3}\right)$ single crystal (Figure S1). ${ }^{10-113}$ A thin mica sheet, back coated with a semitransparent silver mirror, serves as a nonstationary (moving horizontal/lateral) surface. For mechanical support, the mica sheet is glued on a glass cylinder (radius of curvature $10 \mathrm{~mm}$, apex length $10 \mathrm{~mm}$ ) by epoxy resin. Preparation procedures and material parameters for both surfaces are provided in the Supporting Information (SI).
The incident X-ray beam enters through the side of the upper substrate in the direction of the cylinder apex. This avoids scattering artifacts from the mica edges. The $\mathrm{Al}_{2} \mathrm{O}_{3}$ single crystal reduces background scattering from the substrate. Because in reflection geometry the beam has to penetrate several millimeters of $\mathrm{Al}_{2} \mathrm{O}_{3}$, high-energy X-rays are essential. ${ }^{114-116}$

Because of the elasticity of the hardened epoxy resin, the mica sheet can adapt to the planar nonstationary surface when pressed against each other. This generates a slit pore with length $l=4.8 \mathrm{~mm}$ defined by the crystal size and width $w \approx 80 \mu \mathrm{m}$ depending on the applied pressure. Thus, the cylinder-on-flat geometry provides a confined area that is much larger than for conventional crossedcylinder setups (Figure 2d). In in-plane scattering geometry (Figure $2 \mathrm{~b}$ ), the $\mathrm{X}$-ray beam illuminates a sample volume of $10^{-3} \mathrm{~mm}^{3}$ at a slit-pore gap width of $D \approx 2.5 \mu \mathrm{m}$. This volume is about 10 to 50 times larger compared to that of other X-SFA setups in reflection geometry, ${ }^{88,92}$ with the caveat of difficult alignment.

The SFA is mounted inside of a cylindrical gastight stainless steel chamber (Figure 3). A helium atmosphere increases the thermal stability of the setup $\left( \pm 0.05{ }^{\circ} \mathrm{C}\right.$ during an $8 \mathrm{~h}$ shift, $\pm 0.01{ }^{\circ} \mathrm{C}$ during a $15 \mathrm{~min}$ experiment) by improving heat dissipation from the visible light absorbed in the sample. Moreover, helium reduces the scattering background. The relative humidity (r.h.) inside the chamber can be controlled to between 0 and $90 \%$ with $\pm 3 \%$ stability by a humidifier inserted in the helium stream, constantly purging the chamber.

The alignment of the nanometer confinement in cylinderon-flat geometry is measured and controlled using an interference microscope based on multiple-beam white-light interferometry (Figure 4b). A goniometer is used to orient the apex of the cylindrical disc in parallel with the flat surface. Successful alignment is characterized by the shape of the interference pattern (Newton's rings) in the transmitted beam (Figure 4a). By tilting, the interference pattern is optimized from parabolic curves to parallel straight lines. To achieve such alignment over the entire area confined by the opposing interfaces, mica surfaces are glued to a cylindrical support to avoid wrinkling. To provide adequate stiffness, mica sheets are ideally 8 to $10 \mu \mathrm{m}$ thick.

Micrometer screws are used to manually adjust the surfaces to a few micrometers of separation. For dynamic studies, the cylindrical surface can be moved normally and laterally against the stationary planar surface with nanometer precision using piezoelectric transducers. Horizontal sliding motion is aligned in the direction of the cylindrical contact. This maintains the confined area in the rotation center of the diffractometer and the $\mathrm{X}$-ray beam. Strain gauges are used for simultaneously recording forces in both lateral and normal directions.

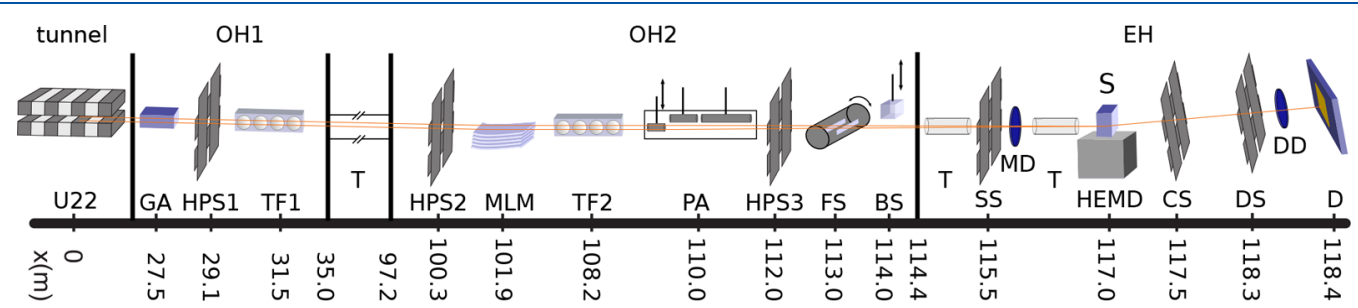

Figure 5. Beamline setup for high-energy scattering experiments at ID31 ESRF (Feb 2016): (OH1/2) optics hutches; (EH) experimental hutch; (U22) $22 \mathrm{~mm}$ periode permanent magnet in-vacuum undulator; (GA) gas absorber ( $1 \mathrm{~m}$ argon at $300 \mathrm{mbar})$; (HPS1/2/3) high power slits; (TF1/2) compound refractive lens (CRL) transfocators; (MLM) horizontal multilayer double monochromator in fixed-exit geometry; (PA) PEEK (polyether ether ketone) absorber set; (FS) rotary fast shutter; (BS) beam safety shutter; (T) flight tubes; (SS) secondary slits; (MD) silicon PIN monitor diode; (HEMD) high-energy microdiffraction setup; (S) sample position; (CS) collimator slits; (DS) detector slits; (DD) silicon PIN detector diode; and (D) 2D detector (CdTe MAXIPIX). Distances are not to scale. 
Figure S3 displays typical force versus distance and force versus time characteristics recorded with the X-SFA in slit-pore geometry. The maximum applied pressure is determined by the chosen combination of the piezo travel range, cantilever spring constant, and elasticity of the glue. This limits the recording of constant-approach-rate force-distance characteristics to approximately $D=200 \mathrm{~nm}$. For highly viscous liquids, smaller gap widths can be achieved by applying periodic compression/ decompression cycles (Figure 10a). Using this technique, a minimum distance of $110 \mathrm{~nm}$ corresponding to less than 40 smectic 8 CB layers was reached. For water, a $7 \mathrm{~nm}$ confinement can be prepared at comparable compression forces using the same components.

In-Situ X-ray Scattering and Reflectivity. X-ray scattering experiments have been performed at the high-energy beamline for buried interface structure and materials processing ID31 at the ESRF-European Synchrotron Radiation Facility, Grenoble, France. Figure 5 shows a sketch of the beamline layout and X-ray optics. The X-ray beam from a cpmU22 undulator is parallelized by the first CRL transfocator $(\mathrm{TF} 1)^{117,118}$ and monochromized by two multilayer mirrors (MLM, energy $70.0 \mathrm{keV}$ ). A second CRL transfocator (TF2) focuses the beam onto the sample position with a $5 \times 20 \mu \mathrm{m}^{2}$ spot size normal and parallel to the slit pore, respectively. To minimize the radiation dose on the sample, a seven-stage absorber (poly(methyl methacrylate), PA) and fast shutter (FS) are used.

For sample positioning and orientation, the X-SFA was mounted onto the six-circle high-energy microdiffractometer (HEMD) setup for surface and interface studies. ${ }^{115,116}$ Scattering intensities are alternatively detected by two $2 \mathrm{D}$ hybride pixel detectors. For XRR experiments requiring the detection of scattering and XRR signals with low background, a CdTe MAXIPIX system (256 pixels $\times 256$ pixels, $55 \mu \mathrm{m}$ pixel size) is mounted on the HEMD detector stage behind a collimation system (CS, DS). The Dectris PILATUS $3 \mathrm{X}$ CdTe $2 \mathrm{M}$ detector (1478 pixels $\times$ 1679 pixels, $172 \mu \mathrm{m}$ pixel size), behind a beam stop (not shown) to absorb the primary and specular reflected beam, is mounted on a dolmen-like granite construction ${ }^{119}$ to cover a wide $q$ range.

Sample degradation is a common problem in experiments using high-brillant synchrotron radiation. ${ }^{120}$ The absorbed photons affect samples via a complex cascade. ${ }^{121}$ However, this so-called radiation damage is related to the absorbed X-ray dose. For soft matter, the absorbed dose per incident X-ray photon exhibits a minimum between 40 and $80 \mathrm{keV}^{21,122}$ Experiments indicated that near solid/liquid and solid/solid interfaces X-rays absorbed not only by soft matter but also near the hard substrate surface have to be considered. ${ }^{123}$ Therefore, under high energies X-ray illumination soft matter samples near solid interfaces tend to be much more stable compared to X-ray energies below $20 \mathrm{keV}$ where most conventional scattering experiments are performed.

Although references from previous similar work might provide some hints, a quantitative prediction of the sample stability in a given experiment is difficult. For example, silane oils such as tetrakis(trimethylsiloxy)silane, tetrakis(2-ethylhexoxy)silane, decamethylcyclopentasiloxane, and octamethylcyclotetrasiloxane might decompose in the X-ray beam so quickly that time-resolved experiments in confinement could be difficult.

To probe buried interfaces in XRR, the high-energy X-ray beam impinges on the slit pore through the side of the corundum single crystal (Figure S1, length $4.8 \mathrm{~mm}, \mathrm{X}$-ray transmission $68 \%)$. Background scattering from the single-crystalline substrates is primarily caused by Compton and thermal diffuse scattering and is significantly reduced compared to that of amorphous materials. At the critical angle $q_{\mathrm{c}}=0.77 \mathrm{~nm}^{-1}$ of gold, the footprint on the substrate is $4.8 \mathrm{~mm}$.

Density profiles perpendicular to the confining interfaces were investigated by X-ray reflectivity (XRR) (Figure 2c). During XRR experiments $R\left(q_{z}\right)$ with varying incident angle $\alpha$, the entire $\mathrm{X}$-SFA setup is tilted by the HEMD diffractometer. Scattering experiments at $\alpha=0$ and with the momentum transfer $q$ parallel to the slit pore probe the in-plane structure of the confined liquid (Figure $2 \mathrm{~b}$ ). Scattering angles were converted to momentum transfer using $q=\frac{4 \pi}{\lambda} \sin (\theta)$.

\section{STRUCTURE AND DYNAMICS IN CONFINED 8CB}

Smectic Liquid Crystal 8CB. As model system, we choose liquid crystal (LC) 4'-octyl-4-cyano-biphenyl (8CB). Liquid crystal $8 \mathrm{CB}$ has been extensively studied using crossed-cylinder SFAs and X-SFAs in transmission geometry. ${ }^{78-81,124,125}$ Compared to other systems, it exhibits a relatively high stability under intense $\mathrm{X}$-ray illumination and has been shown to be an ideal system for assessing the capabilities of an X-SFA.

At $22^{\circ} \mathrm{C}, 8 \mathrm{CB}$ exhibits a smectic A ( SmA) phase (Figure 6). $8 \mathrm{CB}$ is known to form dimers by the $\pi-\pi$ stacking of adjacent

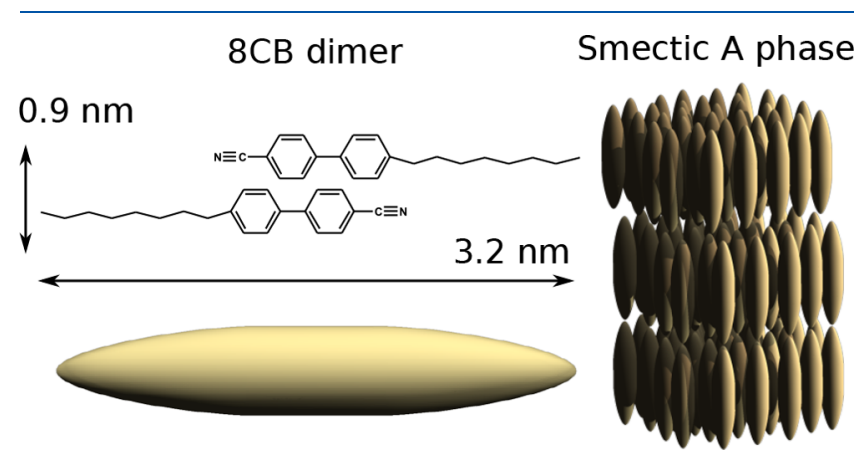

Figure 6. Rod-shaped (calamitic) structure of 4'-octyl-4-cyanobiphenyl (8CB) dimers (left) and molecular alignment in the liquidcrystalline smectic A phase (right).

phenyl rings. This leads to a dimer dimension larger than a single $8 \mathrm{CB}$ molecule (Figure 6). ${ }^{37}$ The lamellar arrangement of the rodshaped $8 \mathrm{CB}$ dimers in smectic layers gives rise to a pronounced scattering peak in the small-angle scattering (SAXS) regime. In $8 \mathrm{CB}$, bulk measurements showed that shear stress induces alignment and a reduction of defects and initiates the growth of smectic grains. ${ }^{107}$ For shorter homologue $6 \mathrm{CB}$, resonance shear measurements in confinement detected an increase in viscosity for $D<20 \mathrm{~nm} .{ }^{126}$ Below $12.5 \pm 1.3 \mathrm{~nm}$, the orientation of the confined molecules was so large and the rigidity was so high that the LC structure could not be distorted by electric fields.

Here, aside from static XRR and in-plane scattering on the LC structure in slit-pore confinement, time-resolved experiments were carried out during compression and decompression cycles. Furthermore, the recorded X-ray scattering and reflectivity data was complemented by simultaneous interferometry-based thickness measurements. Therefore, in contrast to static X-SFA ${ }^{125}$ and macroscopic dynamic experiments, ${ }^{107}$ our setup provides direct access to the structural relaxation dynamics of the confined LC. To study confined structures over a wide gap range, data was taken at $D=1700 \mathrm{~nm}$ and $120 \mathrm{~nm}$ gap widths. For a defined orientation of the $8 \mathrm{CB}$ molecules at the upper and lower interfaces, both confining surfaces were hydrophobized by 
self-assembled monolayers of hexadecane-thiol and octadecyltrichlorosilane, respectively. From work by the Israelachvili group, it is known that in such hydrophobized slit pores the long axis of $8 \mathrm{CB}$ dimers is aligned parallel to the surface normal. ${ }^{37}$

Static Structure of $8 C B$ in Confinement. Figure 7 a shows in-plane scattering data at $D=1700 \mathrm{~nm}$. In in-plane scattering
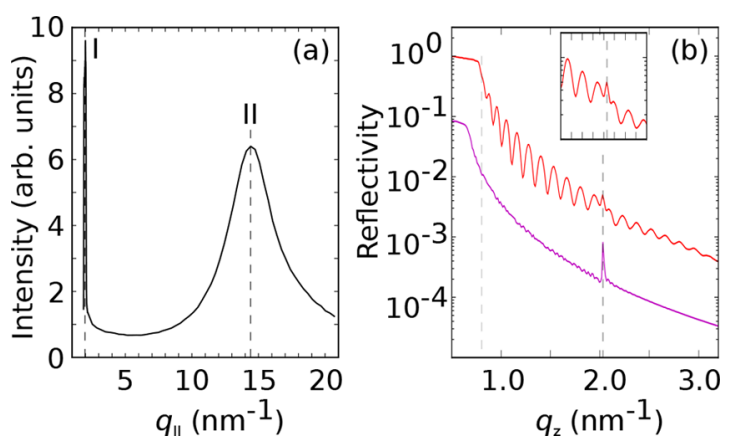

Figure 7. Scattering signal from confined $8 \mathrm{CB}$. (a) In-plane pattern $I\left(q_{\|}\right)$recorded in the scattering geometry depicted in Figure $2 \mathrm{~b}$. (b) Measured (red) specular X-ray reflectivity $R\left(q_{z}\right)$. Model calculated reflectivity curve (purple, vertically shifted by 1 order of magnitude) from a periodic arrangement of 560 smectic $8 \mathrm{CB}$ layers arranged with their long axis perpendicular to the solid/liquid interface.

geometry, experiments recording $I\left(q_{\|}\right)$with momentum transfer $q_{\|}=\sqrt{q_{x}^{2}+q_{y}^{2}}$ are sensitive to the structure in the $x-y$ plane (i.e., parallel to the solid/liquid interface (Figure 2b)).

Qualitatively, the recorded data exhibits the characteristic scattering pattern of a LC in the SmA bulk phase. ${ }^{127,128}$ The sharp diffraction peak (I) at $q_{\mathrm{I}}=2.0 \mathrm{~nm}^{-1}$ corresponds to a $\frac{2 \pi}{q_{\mathrm{I}}}=3.1 \mathrm{~nm}$ real-space distance. This periodicity compares well to the periodicity of $3.2 \mathrm{~nm}$ for the $\mathrm{SmA}$ mesophase of $8 \mathrm{CB}$ confined in between two hydrophobized surfaces as observed in SFA experiments. ${ }^{37}$ Therefore, it is assigned to the long axis of an $8 \mathrm{CB}$ dimer, defining the periodicity of the quasi-long-rangeordered liquid-crystalline smectic layers (Figure 6). Around $q_{\mathrm{II}}=$ $14 \mathrm{~nm}^{-1}$ a second, broad diffuse peak (II) appears. Its $0.45 \mathrm{~nm}$ periodicity corresponds to the average lateral distance between neighboring rod-shaped $8 \mathrm{CB}$ dimers within the same smectic layer. The large FWHM of $4 \mathrm{~nm}^{-1}$ originates from the shortrange order perpendicular to the long axis of the $8 \mathrm{CB}$ dimers.

However, on the quantitative level remarkable differences with respect to nontextured bulk samples are observed. In the bulk, the integrated intensities of the sharp (I) and diffuse (II) peaks are of the same magnitude. In contrast, the recorded scattering pattern (Figure 7a) shows a much larger area under peak II. This is the first indication that the director of the confined LC exhibits a preferred orientation parallel to the interface normal.

Specular reflectivity $I\left(q_{z}\right)$ probes the density profile perpendicular to the solid/liquid interface (Figure $7 \mathrm{~b}$, red curve). The critical angle of total reflection at $0.768 \mathrm{~nm}^{-1}$ is given by the large scattering contrast between the gold mirror and the $\mathrm{Al}_{2} \mathrm{O}_{3}$ block. At higher $q_{z}$, the X-ray beam is transmitted into the gold layer and the $8 \mathrm{CB}$ filled slit pore. Kiessig fringes of periodicity $\Delta q=$ $0.15 \mathrm{~nm}^{-1}$ originate from interference at the $40-\mathrm{nm}$-thick gold layer. At approximately $2 \mathrm{~nm}^{-1}$, a sharp Bragg-like peak is observed. As observed for peak $\mathrm{I}$ in in-plane scattering geometry (Figure 7a), this reflection is attributed to the smectic order (i.e., the dimension of the long axis of $8 \mathrm{CB}$ dimers). For comparison, the purple curve in Figure $7 \mathrm{~b}$ shows a calculated XRR curve based on a simplified model consisting of $1700 \mathrm{~nm} 8 \mathrm{CB}$ (i.e., 560 smectic layers with $3.2 \mathrm{~nm}$ periodicity) adjacent to a semiinfinite gold substrate.

At peak I around $2 \mathrm{~nm}^{-1}$, intensities recorded under the specular condition (i.e., with the momentum transfer perpendicular to the solid/liquid interface ( $z$ direction)) are more than 4 orders of magnitude higher compared to the in-plane scattering along $q_{\|}$(Figure S7). The recorded XRR signal at the peak maximum is about twice the intensity of the interpolated Kiessig fringes originating from the gold layer. Likewise, 2D scattering data show a pronounced maximum in and around the specular direction (Figure 8a,c). The observed angular intensity (a)

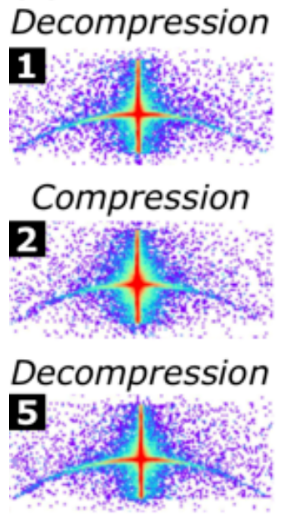

(c)
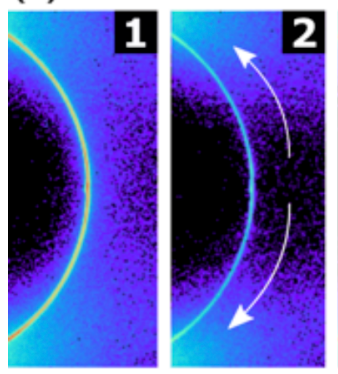

(b)

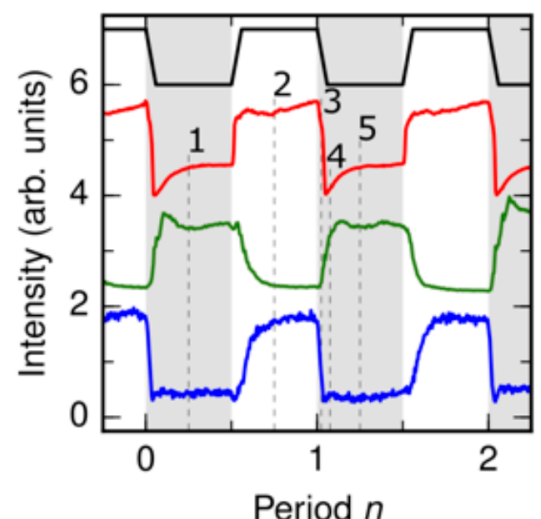

Period $n$
Figure 8. Dynamic compression/decompression experiments at $D \approx$ $1700 \mathrm{~nm}$. (a) Two-dimensional scattering patterns around the specular condition. The arc is located at $q_{\mathrm{I}}=2.0 \mathrm{~nm}^{-1}$. (b) Time evolution of compressive stress (black, $8 \mathrm{~s}$ ramp, $60 \mathrm{~s}$ holding time, $136 \mathrm{~s}$ period, $20 \mathrm{mN}$ force amplitude), the specular XRR SmA first-order signal (red, $q_{\mathrm{I}}=2.0 \mathrm{~nm}^{-1}, q_{z}$ direction), the in-plane-scattering SmA first-order signal (green, $q_{\mathrm{I}}=2.0 \mathrm{~nm}^{-1}, q_{\|}$direction), and the in-plane scattering diffuse signal (blue, $q_{\mathrm{II}}=14 \mathrm{~nm}^{-1}, q_{\|}$direction). Curves are scaled and vertically shifted for clarity. White areas indicate compression, and gray areas, decreased pressure. (c) Two-dimensional pattern in the in-plane direction. The arc is located at $q_{\mathrm{I}}=2.0 \mathrm{~nm}^{-1}$. Areas in the vertical scattering direction are masked to protect the detector from high intensities. Time stamps are numbered 1 to 5 .

distribution over the scattering rings of constant total momentum transfer $q=\sqrt{q_{\|}^{2}+q_{z}^{2}}$ originates from the confinementinduced anisotropic orientation of the LC director. The observed scattering patterns indicate that the smectic $8 \mathrm{CB}$ layers are preferably arranged with their long axis perpendicular to the solid/ liquid interfaces. This layered structure can now be stressed by compression and decompression exerted on the confined LC structure by moving the lower mica cylinder in the vertical direction (inset of Figure 3). 
Relaxation Dynamics. After the experiments at a constant gap width, we now present the structural relaxation dynamics of confined $8 \mathrm{CB}$ that was brought out of equilibrium by periodic compression and decompression cycles. Changes in the gap width $D(t)$ are continuously monitored by the FECO interference pattern. Simultaneously, the arrangement of the $8 \mathrm{CB}$ molecules is probed in real time using specular XRR or X-ray scattering.

At a $D=1700 \mathrm{~nm}$ gap width, confinement corresponds to about 560 smectic layers of $8 \mathrm{CB}$ dimers (Figure 6). A symmetric trapezoidal compression/decompression sequence with an $8 \mathrm{~s}$ ramp and a $60 \mathrm{~s}$ hold time (136 s period) was applied to the confined liquid. Figure $8 \mathrm{~b}$ shows the time evolution over two consecutive periodic cycles. The topmost black curve depicts the compression/decompression process. Gray shaded areas indicate decompression, and white areas, compression. The red curve shows the specular XRR signal at $q_{\mathrm{I}}=2.0 \mathrm{~nm}^{-1}$ with the momentum transfer pointing in the $z$ direction (Figure $7 \mathrm{~b}$ ). Green and blue curves show the in-plane ( $x-y$ plane, Figure $7 \mathrm{a})$ scattering intensities at momentum transfers of $q_{\mathrm{I}}=2.0 \mathrm{~nm}^{-1}$ (peak I) and $q_{\mathrm{II}}=14 \mathrm{~nm}^{-1}$ (peak II), respectively. XRR and scattering data were extracted from the 2D MAXIPIX data sets by integration over a region of interest of $10 \times 10$ detector pixels (solid angle $1.5 \times 10^{-7} \mathrm{sr}$ ).

All recorded signal intensities exhibit a strong periodic time dependence following the applied stress. At $t=0 \mathrm{~s}$, the confinement gap is opening. During the $8 \mathrm{~s}$ decompression intervals, the specular intensity drops below 60\% (Figure S7, red curve). At the same time, the in-plane scattering intensity of peak I is increasing by a factor of 5 (Figure 57, green curve) whereas that of peak II is decreasing below $85 \%$ (Figure S7, blue curve). Upon subsequent compression $(t=68 \mathrm{~s})$, the opposite behavior is observed. While XRR and in-plane peak II intensities are increasing, the in-plane peak I signal decreases. This indicates that upon compression/decompression the signal modulation is not dominated by the amount of $8 \mathrm{CB}$ inside the slit pore. Instead, scattering intensities are redistributed over rings of constant momentum transfer.

More insight into the redistribution of scattering intensities is obtained from the 2D scattering patterns. Figure 8 shows a selection of $2 \mathrm{D}$ detector images recorded around the specular condition (Figure 8a) and in the in-plane direction (Figure 8c). During compression intervals (time stamp 2 and white areas), we observe a sharp specular peak. Low intensity is found in the perpendicular in-plane direction. For decompression segments (time stamps 1 and 5), the situation is reversed. Around the decreasing specular peak, tails emerge along the diffraction ring. Moreover, the scattering intensity is redistributed in offspecular directions where signal intensities increase (Figure S6). The intensity of broad scattering peak II at $q_{\|}=14 \mathrm{~nm}^{-1}$ in the in-plane direction (blue curve) shows inverse signal modulation. This is readily explained by the local anisotropy in the SmA phase of the rod-shaped $8 \mathrm{CB}$ dimers. Here, the long period exhibiting quasilong-range order (low $q$, peak I) is oriented vertically. Perpendicular to this long axis, we have liquidlike short-range order with shorter periodicity (high $q$, peak II).

The observed redistribution of scattering intensities between specular and in-plane directions indicates that the degree of orientational order is strongly affected by compression and decompression (Figure 9). LC domains not aligned in the preferred orientation with respect to the interfaces possess grain boundaries with respect to the aligned majority domain. Grain boundary energy makes these misaligned domains energetically unstable.

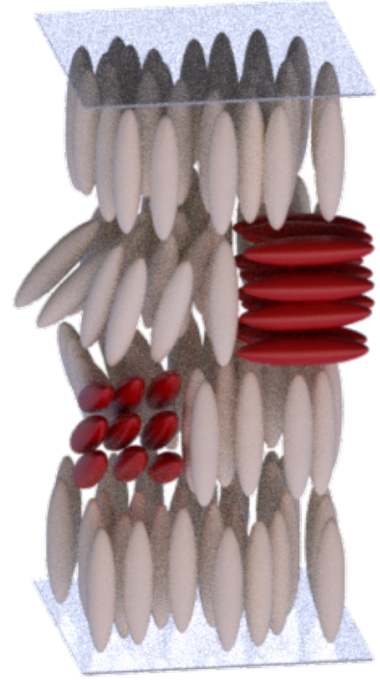

Figure 9. Sketch of the molecular structure of confined $8 \mathrm{CB}$. The functionalized solid surfaces (blue) induce an orientation with the LC director parallel to the interface normal (brown). Grain boundary energies make misaligned domains (red) energetically unstable. However, they can gradually adjust in height during slit-pore opening. During compression, these $8 \mathrm{CB}$ dimers get integrated into the majority domain (brown), leading to an overall increase in alignment.

However, under static conditions, misaligned domains can be kinetically trapped. During compression, material is squeezed in the $x$ and $y$ directions out of the gap. This flow induces a dynamic into the system that helps to overcome energy barriers for defect migration. In particular, the layer structure of misaligned domains with the director pointing in the flow direction will be distorted by shear. In contrast, layers with their normal parallel $z$ direction remain intact and accommodate the $8 \mathrm{CB}$ dimers of the reducing misaligned domains. Therefore, the overall alignment of smectic layers parallel to the confining interfaces increases.

During decompression, $8 \mathrm{CB}$ dimers leave the interface-aligned smectic layers to fill the extra space. Newly developing domains with the director perpendicular to the surface normal can adjust their height to the gradually opening slit pore. This explains the strong 5-fold intensity increase of peak I at $q_{\mathrm{I}}=2.0 \mathrm{~nm}^{-1}$ in the in-plane direction (Figure S7, green curve). A similar orientation of $8 \mathrm{CB}$ dimers perpendicular to the majority domain has been observed in simulations. ${ }^{129}$ They belong to so-called parking-lot states that occur as transition states when rod-shaped $8 \mathrm{CB}$ dimers diffuse from one smectic layer to the other.

Further information on the dynamics of structure formation is obtained from the relaxation of the scattering signal following compression and decompression. After decompression is completed, the specular intensity is gradually recovered (Figure $8 \mathrm{~b}$, red curve, time stamps 1 and 5 ). With a relaxation time scale of approximately $20 \mathrm{~s}$, the signal is converging toward a plateau value during the $60 \mathrm{~s}$ holding time. However, a degree of orientational order comparable to what was observed after compression is not regained. Likewise, during compression, clear relaxation processes on comparable time scales were observes for peak I and II in the in-plane direction (Figure $8 b$, green and blue curves, time stamp 2).

The observed time scale is about 8 orders of magnitude slower compared to the molecular relaxation time for intersmectic layer correlations in the bulk. ${ }^{130}$ In liquids confined on the molecular length scale, a slowdown in dynamics by 8 orders 

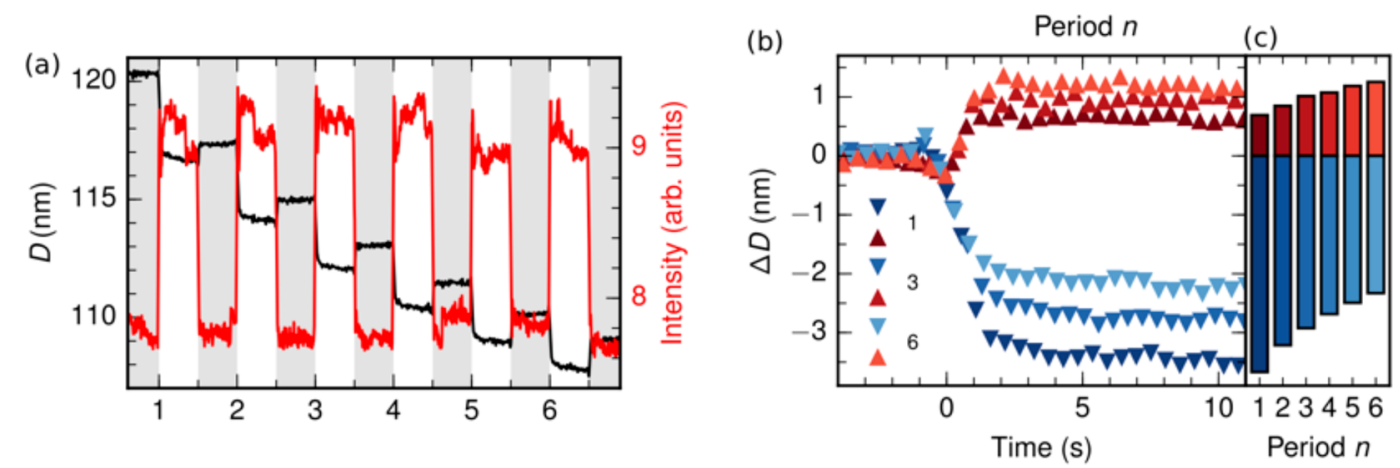

Figure 10. Cyclic compression/decompression experiment at gap width $107 \mathrm{~nm} \leq D \leq 120 \mathrm{~nm}$ (trapezoidal force profile, $20 \mathrm{mN}$ amplitude, $2 \mathrm{~s}$ ramp). (a) Time evolution of the gap distance (black) and normalized specular XRR signal $I\left(q_{z}\right)$ (red) during $64 \mathrm{~s}$ compression/decompression periods. White areas indicate compression, and gray areas, decreased pressure. (b) Relative change in gap width $\Delta D(t)$ during compression (blue triangles down) and decompression (red triangles up) periods $n=1$ (dark), 3 (medium), and 6 (light). (c) Overall step heights $\Delta D(n)$ extracted from gap widths averaged over the last $10 \mathrm{~s}$ of the holding time of subsequent half cycles.

of magnitude was reported. ${ }^{41}$ However, the $1700 \mathrm{~nm}$ slit pore contains about 560 smectic layers. Therefore, we suggest that the observed time scale originates from collective dynamics such as domain reorientation.

After discussing the results obtained for a gap width greater than $1 \mu \mathrm{m}$, we now present experiments at gap sizes below $120 \mathrm{~nm}$. This confinement gap amounts to an equivalent of less than 40 smectic $8 \mathrm{CB}$ layers. At this distance, the gap width can be precisely measured by FECO.

Figure 10a shows the results from an XRR measurement (red curve) recored during periodic compression/decompression cycles. A trapezoidal force profile (amplitude $20 \mathrm{mN}$ ) with a $2 \mathrm{~s}$ ramp, $30 \mathrm{~s}$ holding time, and $64 \mathrm{~s}$ period was applied. Over five full compression/decompression cycles, the gap distance (black curve) decreases by approximately $10 \mathrm{~nm}$. As observed for the $1700 \mathrm{~nm}$ slit pore, compression results in an increase in the specular XRR signal (white areas) while intensities decrease for decompression (gray areas).

During initial compression, the gap size decreases by $\Delta D_{\downarrow}=$ $-3.7 \mathrm{~nm}$ (Figure 10c). This distance is similar to the dimension of $3.2 \mathrm{~nm}$ for the long axis of an $8 \mathrm{CB}$ dimer (peak I). Therefore, we conclude that during initial compression a full smectic $8 \mathrm{CB}$ layer is squeezed out of the slit-pore. However, during decompression the gap opens by only $\Delta D_{\uparrow}=+0.7 \mathrm{~nm}$. This value corresponds to the short axis of an $8 \mathrm{CB}$ dimer (peak II). Accordingly, after decompression, domains with the director perpendicular to the interface normal were observed for the $1700 \mathrm{~nm}$ slit pore.

During subsequent compression events, the change in gap width decreases monotonically to $\Delta D_{\downarrow}=-2.3 \mathrm{~nm}$ (Figure 10b). In contrast, during decompression $\Delta D_{\uparrow}$ increases to $+1.3 \mathrm{~nm}$. Hence, the overall decrease in gap width per compression/ decompression cycle is decreasing with time. During decompression, domains with the long axis lying parallel to the confining surfaces emerge. This indicates the formation of domains that get periodically reoriented during subsequent compression/ decompression cycles.

Unlike for the $1700 \mathrm{~nm}$ slit pore, no clear relaxation processes indicating reorientation are observed by XRR. Likewise, after the $2 \mathrm{~s}$ decompression ramp only minor gap width changes were detected during the subsequent $10 \mathrm{~s}$ holding time (Figure 10b). This indicates that for the small gap width of $120 \mathrm{~nm}$, corresponding to less than 40 smectic $8 \mathrm{CB}$ layers, and on the minute time scale defects are largely trapped. Likewise, Nakano et al. reported a relaxation time on the order of $1 \mathrm{~h}$ for confined $8 \mathrm{CB}$ under pressure. ${ }^{125}$ Therefore, external stimuli are required to overcome energy barriers and induce domain reorientation.

\section{CONCLUSIONS AND OUTLOOK}

X-SFA in Cylinder-on-Flat Geometry. Over the last few decades, several groups presented scientific instruments to investigate the molecular-scale structure of fluids, confined inside a slit pore, by X-ray and neutron scattering techniques. Reviewing the capabilities of the complementary designs, we motivate the need for a novel approach that allows

- the study of the structure of soft matter in slit pores parallel and perpendicular to the confining interfaces by specular XRR and X-ray scattering,

- control of the slit-pore gap distance on the micrometerto-nanometer length scale,

- simultaneous measurements of the normal and tangential forces acting on the confining interfaces while recording X-ray scattering data, and

- an investigation of the relaxation dynamics of a system that was brought out of equilibrium by compression/ decompression or shear stress in time-resolved experiments.

On the basis of geometry considerations, we came up with a novel X-SFA using the cylinder-on-flat geometry. This design can fulfill all of the requirements outlined above in a single instrument. Compared to previous X-SFA designs, the cylinder-on-flat geometry allows us to increase the amount of illuminated confined sample volume by more than 1 order of magnitude. Thus, the described instrument is no longer limited to static measurements. In addition, time-resolved scattering experiments with high temporal resolution become feasible. At the same time, the X-ray dose deposited on the sample is kept at an acceptable level.

An advantage of the new X-SFA instrument is its capability for dynamic in situ studies on the response of a confined liquid after an external stimulus. The system can be brought out of equilibrium by shear stress or compression and decompression of the slit pore. Subsequently, structural relaxation dynamics are recorded by scattering techniques. At the same time, force and distance measurements provide complementary information on the dynamics of the system under sliding conditions. ${ }^{36,37,82}$

The feasibility of the new X-SFA geometry was demonstrated by experiments on the smectic LC $8 \mathrm{CB}$. The X-SFA can be employed to create and control slit-pore confinement on the $10 \mathrm{~nm}$ length scale while simultaneously determining the molecular-scale structure of soft matter by X-ray scattering 
techniques. Furthermore, it enables the probing of structural relaxation dynamics of confined LC mesophases under stress.

Scientific Research Directions. Possible fields for future studies include systems that exhibit anisotropic nonequilibrium structures that are induced by shear forces. Shear forces can induce an orientation in confined liquids and modify their phase stability. For macromolecules, large anisotropic molecules, and LCs, such effects are already well-known. ${ }^{107,126}$ However, compared to classical rheometers, a SFA can provide higher shear rates. This allows studies on confined liquids with lower viscosity and faster dynamics. Examples include water and short-chain alkanes, in which the influence of shear on the interfacial structure is still under debate. $^{104,105,131}$

Moreover, the structure and dynamics of confined liquids play crucial roles in topics ranging from energy materials to environmental sciences. Examples include subjects related to the spreading of surfactants, wetting dynamics, electrolytes in electric double-layer capacitors and batteries, and the structure and dynamics of confined polymer melts. In nanotribology, open questions such as friction and abrasive behavior on the nanometer length scale can be addressed. ${ }^{132}$

A particularly interesting research direction includes studies on confined complex electrolytes such as ionic liquids and complex solvent mixtures. These materials exhibit structural heterogeneities on the nanometer length scale. ${ }^{6,7,9,30,133}$ At solid/liquid ${ }^{25,134,135}$ and liquid/vapor interfaces, ${ }^{19,29,136,137}$ layered structures have been observed. Therefore, it is anticipated that confinement can have strong influences on IL structures $^{138-140}$ and dynamics. ${ }^{141,142}$

Future Instrument Developments. Aside from shear and normal forces (Figure 1e), other stimuli can be employed to drive a confined system out of equilibrium. Examples include the application of an electrical potential (Figure 1f). This provides the ability to investigate the dynamics of interfacial hydration layers and the adsorption and desorption of ions in solution. Another possibility is short light pulses. They can be used to rapidly increase temperature to study phase transitions in confinement. Alternatively, light can be used to switch the conformation of molecules such as azobenzenes. Subsequently, the structural response of the confined system to conformational changes can be investigated.

With the upcoming upgrades of synchrotron radiation sources to diffraction-limited storage rings ${ }^{45,46}$ entirely new types of scattering experiments will become feasible. Currently, typical beam sizes for high-energy X-rays at PETRA III and ESRF are in the range of $10 \mu \mathrm{m}$ horizontally and $5 \mu \mathrm{m}$ vertically. After the ESRF EBS upgrade, the projected beam size at ID31 will be $1 \mu \mathrm{m} \times$ $200 \mathrm{~nm}$ (horizontal $\times$ vertical) at a 15-times higher flux compared to the current flux. For X-SFA experiments, we foresee the potential for two important breakthroughs that are beyond current capabilities.

Currently, typical vertical beam sizes are compatible with the approximately $100-\mu \mathrm{m}$-wide homogeneous slit pores formed by pressing a flexible, supported mica cylinder onto a rigid, flat substrate. However, we anticipate that in the future hard material SFA contacts among metals, semiconductors, and ceramics will also be interesting probe systems with high relevance in industrial applications. Because of their high elastic modulus, these materials cannot adapt to the opposing flat surface. Therefore, it will be challenging to generate slit pores with constant gap thicknesses, laterally extending over more than a few micrometers. Thus, smaller horizontal beam sizes on the order of $1 \mu \mathrm{m}$ will be highly beneficial for studying realistic systems such as wet lubricants between hard matter surfaces.

Second, there is a large amount of interest in the structure and dynamics of liquids confined between laterally heterogeneous interfaces. The heterogeneity can be related to the topography (roughness), surface functionalization (hydrophilic vs hydrophobic), or surface charge (Figure 11). One of the

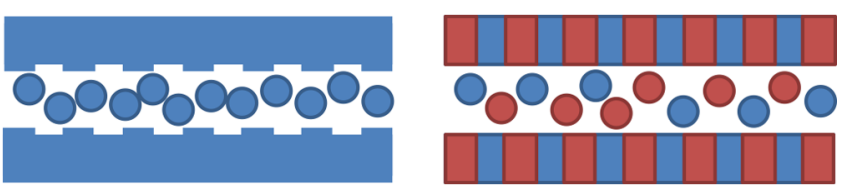

Figure 11. Smaller vertical beam sizes, available at upcoming diffractionlimited storage rings, will enable spatially resolved experiments on laterally inhomogeneous slit pores. Examples include engineered surface topographies mimicking roughness (left) and hydrophilic vs hydrophobic or negatively vs positively charged surface patterns (right).

motivations is to understand the arrangement and mobility of ions in microporous materials composed of hydrophilic and hydrophobic moieties. Thus, smaller horizontal beam sizes will open up new experimental possibilities to answer these kinds of scientific questions.

\section{ASSOCIATED CONTENT}

\section{S Supporting Information}

The Supporting Information is available free of charge on the ACS Publications website at DOI: 10.1021/acs.langmuir.9b01215.

Experimental description of surface preparation and characterization, material properties, technical details of the X-SFA setup and operation, and complementary $8 \mathrm{CB}$ scattering data (PDF)

\section{AUTHOR INFORMATION}

\section{Corresponding Authors}

*E-mail: valtiner@iap.tuwien.ac.at.

*E-mail: mezger@mpip-mainz.mpg.de.

ORCID $\odot$

Frank Uwe Renner: 0000-0003-0425-393X

Markus Mezger: 0000-0001-9049-6983

Notes

The authors declare no competing financial interest.

Biographies

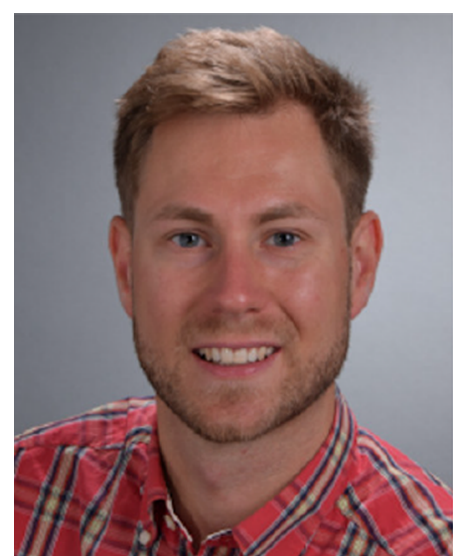

Henning Weiss received his Ph.D. in Markus Mezger's group in 2018 with a thesis titled "Structure of Confined Liquids Studied by an X-ray 
Surface Force Apparatus". Currently he is working as a IT consultant at Bilfinger Digital Next.

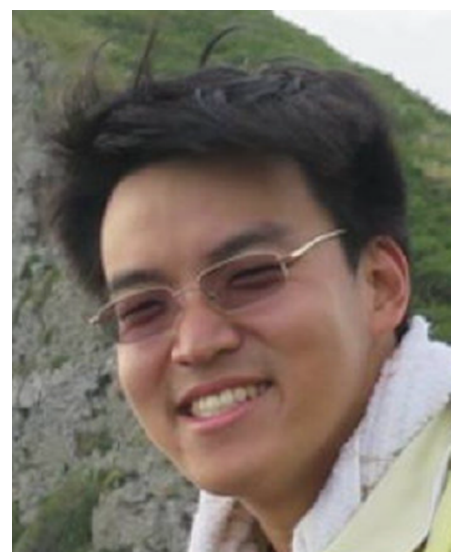

Hsiu-Wei Cheng is university assistant in the applied interface physics group at the Vienna University of Technology (TU Wien). He received his Ph.D. from Ruhr University Bochum in 2017, and then he was postdoctoral researcher at the Freiberg University of Technology.

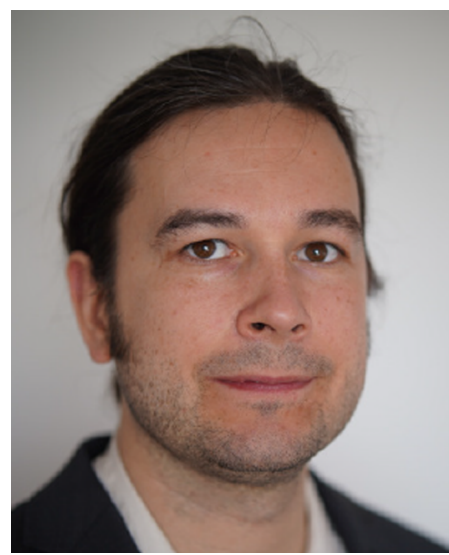

Julian Mars received his Ph.D. in Markus Mezger's group in 2019 with a thesis titled "Microphase-Separated Ionic Liquid-Organic Compound Binary Solutions-Bulk Structure and Surface Order”. Currently he is a postdoctoral researcher at Stanford Synchrotron Radiation Lightsource, Menlo Park.

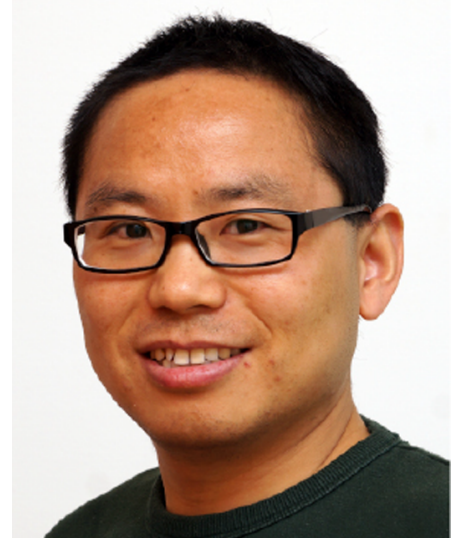

Hailong Li received his Ph.D. in Markus Mezger's group in 2017 with a thesis titled "Interfacial Premelting of Ice in Nanocomposite Materials". Currently he is a postdoctoral researcher in the Department of Fibre and Polymer Technology at KTH Royal Institute of Technology, Stockholm.

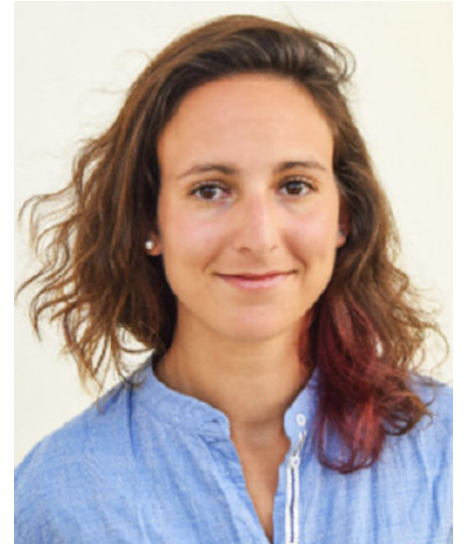

Claudia Merola received her Ph.D. in Markus Valtiner's group in 2019 with a thesis titled "Unravelling the Corrosion Mechanism in Nanometer Confined Gaps Using White Light Interferometry in Reflection and Transmission Mode".

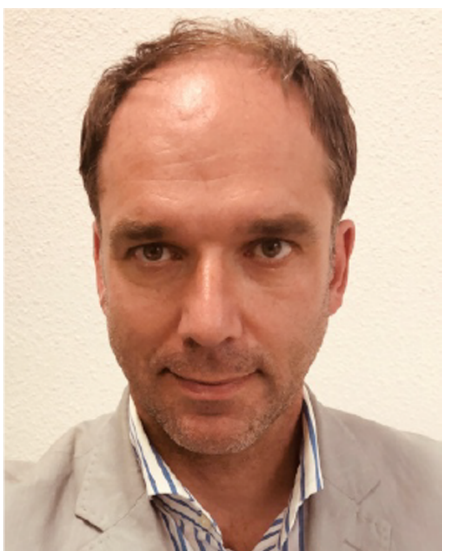

Frank Uwe Renner is a professor of energy materials and physics at the Institute for Materials Science of Hasselt University in Belgium and the joint research center IMOMEC of Hasselt University and IMEC. After obtaining a doctorate degree from University of Stuttgart in Germany, he pursued postdoctoral research at the European Synchrotron Radiation Facility in Grenoble, France, and at the Research Institute for Ubiquitous Energy Devices at AIST Ikeda in Japan with an Alexander-von-Humboldt stipend. From 2007 to 2013, he was a research group leader at the Max-Planck-Institute for Iron Research in Düsseldorf, Germany. He uses synchrotron light and is involved with APT-Flanders and the installation of a laboratory-based HAXPES setup at Hasselt University. His scientific interests center around surface science and electrochemistry, including the mechanistic understanding of corrosion processes, Li-ion battery electrodes and interfaces, and also biorelated lipid membranes.

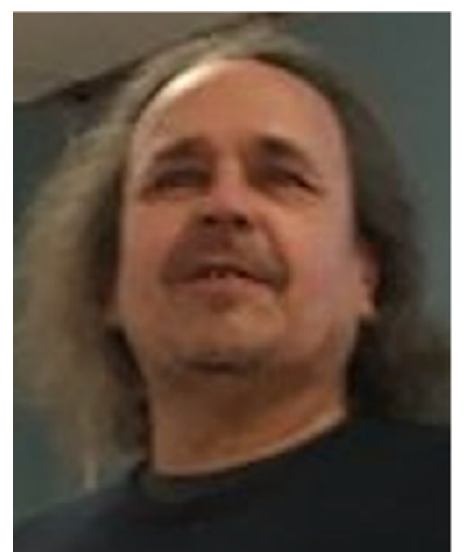

Veijo Honkimäki is the head of the Structure of Materials group at ESRF. He is also in charge of high-energy beamline ID31 for "Buried Interface Structure and Materials Processing". 


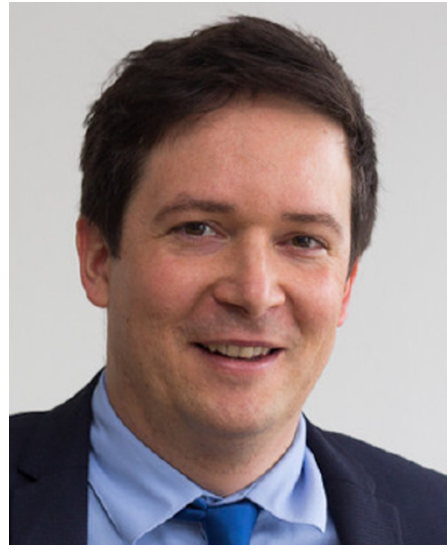

Markus Valtiner is a professor of applied interface physics at the Vienna University of Technology (TU Wien). He received his Ph.D. in 2008 for his graduate work at the Max-Planck-Institut für Eisenforschung (MPI-E) in Düsseldorf, Germany. From 2009 to 2012, he worked as postdoctoral researcher in the Department of Chemical Engineering, University of California, Santa Barbara. In 2012, he again joined the MPI-E as a group leader, and from 2016 to 2017, he was a professor of physical chemistry at the Freiberg University of Technology. His scientific interests are focused on solid/liquid interfaces, singlemolecule interactions, and adhesion as well as corrosion in confined spaces using force probe experiments and, in particular, the surfaces forces apparatus.

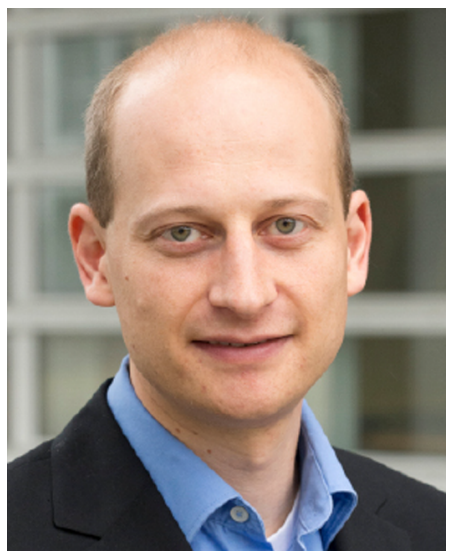

Markus Mezger is a group leader at the Max Planck Institute for Polymer Research (MPI-P) and a professor at the Institute of Physics, Johannes Gutenberg University, Mainz, Germany. He received his Ph.D. in physics in 2008 from the University of Stuttgart. After postdoctoral research at the Department of Chemical Engineering, University of California, Berkeley, and at Lawrence Berkeley National Laboratory, he joined MPI-P in 2010. His scientific interests are focused on the structure and dynamics of soft matter at interfaces and in confinement as studied by X-ray and neutron scattering techniques. Current research topics address ionic liquids, electrolyte solutions, water, ice, and polymers.

\section{ACKNOWLEDGMENTS}

The authors acknowledge the help of Thomas Buslaps (ESRF) at beamline ID31, Marie Ruat (ESRF) for support on the CdTe MAXIPIX detector, and Michael Kappl (MPI-P) for AFM measurements and thank Harald Reichert (ESRF), Milena Lippmann (PETRA III), and Hans-Jürgen Butt (MPI-P) for helpful discussions. J.M. and M.M. acknowledge the MAINZ Graduate School of Excellence, funded through the Excellence Initiative (DFG/GSC 266). M.V. was supported by an ERC
Starting Grant (no. 677663), and H.L., by the China Scholarship Council.

\section{REFERENCES}

(1) Simon, P.; Gogotsi, Y. Materials for electrochemical capacitors. Nat. Mater. 2008, 7, 845-854.

(2) Fedorov, M. V.; Kornyshev, A. A. Ionic Liquids at Electrified Interfaces. Chem. Rev. 2014, 114, 2978-3036.

(3) Kristiansen, K.; Valtiner, M.; Greene, G. W.; Boles, J. R.; Israelachvili, J. N. Pressure solution-The importance of the electrochemical surface potentials. Geochim. Cosmochim. Acta 2011, 75, 68826892.

(4) Frackowiak, E.; Beguin, F. Carbon materials for the electrochemical storage of energy in capacitors. Carbon 2001, 39, 937-950.

(5) Steinrueck, H.-P.; Wasserscheid, P. Ionic Liquids in Catalysis. Catal. Lett. 2015, 145, 380-397.

(6) Zemb, T.; Klossek, M.; Lopian, T.; Marcus, J.; Schöttl, S.; Horinek, D.; Prevost, S.; Touraud, D.; Diat, O.; Marcelja, S.; Kunz, W. How to explain microemulsions formed by solvent mixtures without conventional surfactants. Proc. Natl. Acad. Sci. U. S. A. 2016, 113, 4260.

(7) Bier, M.; Mars, J.; Li, H.; Mezger, M. Salt-induced microheterogeneities in binary liquid mixtures. Phys. Rev. E: Stat. Phys., Plasmas, Fluids, Relat. Interdiscip. Top. 2017, 96, 022603.

(8) Holloczki, O.; Berkessel, A.; Mars, J.; Mezger, M.; Wiebe, A.; Waldvogel, S.; Kirchner, B. The Catalytic Effect of Fluoroalcohol Mixtures Depends on Domain Formation. ACS Catal. 2017, 7, 1846.

(9) Weiss, H.; Mars, J.; Li, H.; Kircher, G.; Ivanova, O.; Feoktystov, A.; Soltwedel, O.; Bier, M.; Mezger, M. Mesoscopic Correlation Functions in Heterogeneous Ionic Liquids. J. Phys. Chem. B 2017, 121, 620.

(10) Perkin, S.; Klein, J. Soft matter under confinement. Soft Matter 2013, 9, 10438-10441.

(11) Klein, J.; Kumacheva, E. Confinement-Induced Phase Transitions in Simple Liquids. Science 1995, 269, 816.

(12) Demirel, A. L.; Granick, S. Glasslike transition of a confined simple fluid. Phys. Rev. Lett. 1996, 77, 2261.

(13) Israelachvili, J. N.; Mcguiggan, P. M.; Homola, A. M. Dynamic Properties of Molecularly Thin Liquid Films. Science 1988, 240, 189.

(14) Mezger, M.; Reichert, H.; Schöder, S.; Okasinski, J.; Schröder, H.; Dosch, H.; Palms, D.; Ralston, J.; Honkimäki, V. High-resolution in situ $\mathrm{x}$-ray study of the hydrophobic gap at the water-octadecyltrichlorosilane interface. Proc. Natl. Acad. Sci. U. S. A. 2006, 103, 18401-18404.

(15) Steinrueck, H.-G.; Schiener, A.; Schindler, T.; Will, J.; Magerl, A.; Konovalov, O.; Li Destri, G.; Seeck, O. H.; Mezger, M.; Haddad, J.; Deutsch, M.; Checco, A.; Ocko, B. M. Nanoscale Structure of Si/SiO2/ Organics Interfaces. ACS Nano 2014, 8, 12676-12681.

(16) Mezger, M.; Sedlmeier, F.; Horinek, D.; Reichert, H.; Pontoni, D.; Dosch, H. On the Origin of the Hydrophobic Water Gap: An X-ray Reflectivity and MD Simulation Study. J. Am. Chem. Soc. 2010, 132, 6735-6741.

(17) Ocko, B. Smectic-layer growth at solid interfaces. Phys. Rev. Lett. $1990,64,2160$.

(18) Ocko, B.; Hlaing, H.; Jepsen, P.; Kewalramani, S.; Tkachenko, A.; Pontoni, D.; Reichert, H.; Deutsch, M. Unifying interfacial selfassembly and surface freezing. Phys. Rev. Lett. 2011, 106, 137801.

(19) Mars, J.; Hou, B.; Weiss, H.; Li, H.; Konovalov, O.; Festersen, S.; Murphy, B. M.; Rütt, U.; Bier, M.; Mezger, M. Surface induced smectic order in ionic liquids - an X-ray reflectivity study of C22C1imNTf2. Phys. Chem. Chem. Phys. 2017, 19, 26651-26661.

(20) Lied, A.; Dosch, H.; Bilgram, J. H. Surface Melting of Ice $I_{h}$ Single Crystals Revealed by Glancing Angle X-ray Scattering. Phys. Rev. Lett. 1994, 72, 3554-3557.

(21) Mezger, M.; Schöder, S.; Reichert, H.; Schröder, H.; Okasinski, J.; Honkimäki, V.; Ralston, J.; Bilgram, J.; Roth, R.; Dosch, H. Water and Ice in contact with Octadecyl-Trichlorosilane Functionalized Surfaces: A High Resolution X-ray Reflectivity Study. J. Chem. Phys. 2008, 128, 244705. 
(22) Sanchez, M. A.; et al. Experimental and theoretical evidence for bilayer-by-bilayer surface melting of crystalline ice. Proc. Natl. Acad. Sci. U. S. A. 2017, 114, 227-232.

(23) Nagata, Y.; Hama, T.; Backus, E. H. G.; Mezger, M.; Bonn, D.; Bonn, M.; Sazaki, G. The Surface of Ice under Equilibrium and Nonequilibrium Conditions. Acc. Chem. Res. 2019, 52, 1006-1015.

(24) Huisman, W. J.; Peters, J. F.; Zwanenburg, M. J.; de Vries, S. A.; Derry, T. E.; Abernathy, D.; van der Veen, J. F. Layering of a liquid metal in contact with a hard wall. Nature 1997, 390, 379-381.

(25) Mezger, M.; Schröder, H.; Reichert, H.; Sebastian Schramm, J. S. O.; Schöder, S.; Honkimäki, V.; Deutsch, M.; Ocko, B. M.; Ralston, J.; Rohwerder, M.; Stratmann, M.; Dosch, H. Molecular Layering of Fluorinated Ionic Liquids at a Charged Sapphire (0001) Surface. Science 2008, 322, 424.

(26) Tamam, L.; Pontoni, D.; Hofmann, T.; Ocko, B. M.; Reichert, H.; Deutsch, M. Atomic-Scale Structure of a Liquid Metal-Insulator Interface. J. Phys. Chem. Lett. 2010, 1, 1041-1045.

(27) Mezger, M.; Schramm, S.; Schröder, H.; Reichert, H.; Deutsch, M.; De Souza, E. J.; Okasinski, J. S.; Ocko, B. M.; Honkimäki, V.; Dosch, $\mathrm{H}$. Layering of [BMIM][sup + ]-based ionic liquids at a charged sapphire interface. J. Chem. Phys. 2009, 131, 094701.

(28) Valtiner, M.; Banquy, X.; Kristiansen, K.; Greene, G. W.; Israelachvili, J. N. The Electrochemical Surface Forces Apparatus: The Effect of Surface Roughness, Electrostatic Surface Potentials, and Anodic Oxide Growth on Interaction Forces, and Friction between Dissimilar Surfaces in Aqueous Solutions. Langmuir 2012, 28, 1308013093.

(29) Mezger, M.; Ocko, B. M.; Reichert, H.; Deutsch, M. Surface layering and melting in an ionic liquid studied by resonant soft $\mathrm{X}$-ray reflectivity. Proc. Natl. Acad. Sci. U. S. A. 2013, 110, 3733.

(30) Cheng, H.-W.; Weiss, H.; Stock, P.; Chen, Y.-J.; Reinecke, C. R.; Dienemann, J.-N.; Mezger, M.; Valtiner, M. Effect of Concentration on the Interfacial and Bulk Structure of Ionic Liquids in Aqueous Solution. Langmuir 2018, 34, 2637.

(31) Kind, M.; Peukert, W.; Rehage, H.; Schuchmann, H. P. Colloid Process Engineering; Springer, 2015.

(32) Mezger, M.; Jerome, B.; Kortright, J. B.; Valvidares, M.; Gullikson, E. M.; Giglia, A.; Mahne, N.; Nannarone, S. Molecular orientation in soft matter thin films studied by resonant soft $\mathrm{x}$-ray reflectivity. Phys. Rev. B: Condens. Matter Mater. Phys. 201 1, 83, 155406.

(33) Reichert, H.; Klein, O.; Dosch, H.; Denk, M.; V, H.; T, L.; G, R. Observation of five-fold local symmetry in liquid lead. Nature 2000, 408, 839.

(34) Li, H.; Bier, M.; Mars, J.; Weiss, H.; Dippel, A.-C.; Gutowski, O.; Honkimäki, V.; Mezger, M. Interfacial premelting of ice in nano composite materials. Phys. Chem. Chem. Phys. 2019, 21, 3734-3741.

(35) Sentker, K.; Zantop, A. W.; Lippmann, M.; Hofmann, T.; Seeck, O. H.; Kityk, A. V.; Yildirim, A.; Schönhals, A.; Mazza, M. G.; Huber, P. Quantized Self-Assembly of Discotic Rings in a Liquid Crystal Confined in Nanopores. Phys. Rev. Lett. 2018, 120, 067801.

(36) Golan, Y.; Martin-Herranz, A.; Li, Y.; Safinya, C. R.; Israelachvili, J. Direct observation of shear-induced orientational phase coexistence in a lyotropic system using a modified X-ray surface forces apparatus. Phys. Rev. Lett. 2001, 86, 1263.

(37) Ruths, M.; Steinberg, S.; Israelachvili, J. N. Effects of Confinement and Shear on the Properties of Thin Films of Thermotropic Liquid Crystal. Langmuir 1996, 12, 6637-6650.

(38) Lohmeier, M.; Vlieg, E. Angle calculations for a six-circle surface X-ray diffractometer. J. Appl. Crystallogr. 1993, 26, 706-716.

(39) Israelachvili, J. Thin film studies using multiple-beam interferometry. J. Colloid Interface Sci. 1973, 44, 259-272.

(40) Schoen, M.; Rhykerd, C. L., Jr; Diestler, D. J.; Cushman, J. H. Shear forces in molecularly thin films. Science 1989, 245, 1223-1226.

(41) Granick, S. Motions and relaxations of confined liquids. Science 1991, 253, 1374-1379.

(42) Ducker, W. A. Direct measurement of colloidal forces using an atomic force microscope. Nature 1991, 353, 239-241.
(43) Butt, H.-J. Measuring electrostatic, van der Waals, and hydration forces in electrolyte solutions with an atomic force microscope. Biophys. J. 1991, 60, 1438-1444.

(44) Butt, H.-J.; Cappella, B.; Kappl, M. Force measurements with the atomic force microscope: Technique, interpretation and applications. Surf. Sci. Rep. 2005, 59, 1-152.

(45) Schroer, C. G.; Agapov, I.; Brefeld, W.; Brinkmann, R.; Chae, Y.C.; Chao, H.-C.; Eriksson, M.; Keil, J.; Nuel Gavaldà, X.; Röhlsberger, R.; Seeck, O. H.; Sprung, M.; Tischer, M.; Wanzenberg, R.; Weckert, E. PETRA IV: the ultralow-emittance source project at DESY. J. Synchrotron Radiat. 2018, 25, 1277-1290.

(46) Raimondi, P.; Einfeld, D.; Farvacque, L.; Le Bec, G.; Biasci, J.-C.; Bouteille, J.-F.; Jacob, J.; Scheidt, K.; Chavanne, J.; Perron, T.; White, S.; Hahn, M.; Martin, D. EBS Storage Ring Technical Report; ESRF - The European Synchrotron, 2018.

(47) Israelachvili, J.; Min, Y.; Akbulut, M.; Alig, A.; Carver, G.; Greene, W.; Kristiansen, K.; Meyer, E.; Pesika, N.; Rosenberg, K.; Zeng, $\mathrm{H}$. Recent advances in the surface forces apparatus (SFA) technique. Rep. Prog. Phys. 2010, 73, 036601.

(48) Kienle, D. F.; Kuhl, T. L. Density and Phase State of a Confined Nonpolar Fluid. Phys. Rev. Lett. 2016, 117, 036101.

(49) Heuberger, M.; Zach, M.; Spencer, N. Density fluctuations under confinement: When is a fluid not a fluid? Science 2001, 292, 905-908.

(50) Kittelmann, M.; Rahe, P.; Kühnle, A. Molecular self-assembly on an insulating surface: interplay between substrate templating and intermolecular interactions. J. Phys.: Condens. Matter 2012, 24, 354007.

(51) Kuhn, S.; Rahe, P. Discriminating short-range from van der Waals forces using total force data in noncontact atomic force microscopy. Phys. Rev. B: Condens. Matter Mater. Phys. 2014, 89, 235417.

(52) Marutschke, C.; Walters, D.; Cleveland, J.; Hermes, I.; Bechstein, R.; Kühnle, A. Three-dimensional hydration layer mapping on the (10.4) surface of calcite using amplitude modulation atomic force microscopy. Nanotechnology 2014, 25, 335703.

(53) Söngen, H.; Marutschke, C.; Spijker, P.; Holmgren, E.; Hermes, I. M.; Bechstein, R.; Klassen, S.; Tracey, J.; Foster, A. S.; Kühnle, A. Chemical Identification at the Solid-Liquid Interface. Langmuir 2017, 33, 125.

(54) Seeck, O. H.; Murphy, B. X-ray Diffraction: Modern Experimental Techniques; Pan Stanford, 2015.

(55) Willmott, P. An Introduction to Synchrotron Radiation: Techniques and Applications; John Wiley \& Sons, 2011.

(56) Ezquerra, T. A.; Garcia-Gutierrez, M. C.; Nogales, A.; Gomez, M. Applications of Synchrotron Light to Scattering and Diffraction in Materials and Life Sciences; Springer, 2009; Vol. 776.

(57) Ocko, B.; Wang, J.; Davenport, A.; Isaacs, H. In situ x-ray reflectivity and diffraction studies of the $\mathrm{Au}(001)$ reconstruction in an electrochemical cell. Phys. Rev. Lett. 1990, 65, 1466.

(58) Reichert, P.; Kjær, K. S.; van Driel, T. B.; Mars, J.; Ochsmann, J. W.; Pontoni, D.; Deutsch, M.; Nielsen, M. M.; Mezger, M. Molecular Scale Structure and Dynamics at an Ionic Liquid/Electrode Interface. Faraday Discuss. 2018, 206, 141.

(59) Perakis, F.; et al. Coherent X-rays reveal the influence of cage effects on ultrafast water dynamics. Nat. Commun. 2018, 9, 1917.

(60) Olbinado, M. P.; et al. Ultra high-speed x-ray imaging of laserdriven shock compression using synchrotron light. J. Phys. D: Appl. Phys. 2018, 51, 055601.

(61) Całus, S.; Jabłońska, B.; Busch, M.; Rau, D.; Huber, P.; Kityk, A. V. Paranematic-to-nematic ordering of a binary mixture of rodlike liquid crystals confined in cylindrical nanochannels. Phys. Rev. E 2014, $89,062501$.

(62) Całus, S.; Busch, M.; Kityk, A. V.; Piecek, W.; Huber, P. Chiral phases of a confined cholesteric liquid crystal: anchoring-dependent helical and smectic self-assembly in nanochannels. J. Phys. Chem. C 2016, 120, 11727-11738.

(63) Kityk, A. V.; Huber, P. Thermotropic nematic and smectic order in silica glass nanochannels. Appl. Phys. Lett. 2010, 97, 153124.

(64) Suzuki, Y.; Duran, H.; Steinhart, M.; Butt, H.-J.; Floudas, G. Homogeneous crystallization and local dynamics of poly (ethylene 
oxide)(PEO) confined to nanoporous alumina. Soft Matter 2013, 9, 2621-2628.

(65) Yao, Y.; Sakai, T.; Steinhart, M.; Butt, H.-J.; Floudas, G. Effect of Poly (ethylene oxide) Architecture on the Bulk and Confined Crystallization within Nanoporous Alumina. Macromolecules 2016, 49, 5945-5954.

(66) Lee, W.; Schwirn, K.; Steinhart, M.; Pippel, E.; Scholz, R.; Gösele, $\mathrm{U}$. Structural engineering of nanoporous anodic aluminium oxide by pulse anodization of aluminium. Nat. Nanotechnol. 2008, 3, 234-239.

(67) Schwirn, K.; Lee, W.; Hillebrand, R.; Steinhart, M.; Nielsch, K.; Gösele, U. Self-ordered anodic aluminum oxide formed by $\mathrm{H} 2 \mathrm{SO} 4$ hard anodization. ACS Nano 2008, 2, 302-310.

(68) Zhao, L.; Yosef, M.; Steinhart, M.; Göring, P.; Hofmeister, H.; Gösele, U.; Schlecht, S. Porous silicon and alumina as chemically reactive templates for the synthesis of tubes and wires of $\mathrm{SnSe}, \mathrm{Sn}$, and SnO2. Angew. Chem., Int. Ed. 2006, 45, 311-315.

(69) Huber, P. Soft matter in hard confinement: phase transition thermodynamics, structure, texture, diffusion and flow in nanoporous media. J. Phys.: Condens. Matter 2015, 27, 103102.

(70) Lindlar, B.; Kogelbauer, A.; Kooyman, P. J.; Prins, R. Synthesis of large pore silica with a narrow pore size distribution. Microporous Mesoporous Mater. 2001, 44-45, 89-94.

(71) Yildirim, A.; Sentker, K.; Smales, G. J.; Pauw, B. R.; Huber, P.; Schönhals, A. Collective orientational order and phase behavior of a discotic liquid crystal under nanoscale confinement. Nanoscale Advances 2019, 1, 1104-1116.

(72) Suzuki, Y.; Duran, H.; Akram, W.; Steinhart, M.; Floudas, G.; Butt, H.-J. Multiple nucleation events and local dynamics of poly $(\varepsilon-$ caprolactone)(PCL) confined to nanoporous alumina. Soft Matter 2013, 9, 9189-9198.

(73) Franz, C.; Lange, F.; Golitsyn, Y.; Hartmann-Azanza, B.; Steinhart, M.; Krutyeva, M.; Saalwächter, K. Chain Dynamics and Segmental Orientation in Polymer Melts Confined to Nanochannels. Macromolecules 2016, 49, 244-256.

(74) Ndao, M.; Lefort, R.; Cerclier, C.; Busselez, R.; Morineau, D.; Frick, B.; Ollivier, J.; Kityk, A. V.; Huber, P. Molecular dynamics of pyrene based discotic liquid crystals confined in nanopores probed by incoherent quasielastic neutron scattering. RSC $A d v$. 2014, 4, 5935859369.

(75) Thomson, J. J. Applications of Dynamics to Physics and Chemistry; Macmillan, 1888.

(76) Rie, E. Über den Einfluss der Oberflächenspannung auf Schmelzen und Gefrieren. Z. Phys. Chem. 1923, 104, 354-362.

(77) Hansen-Goos, H.; Wettlaufer, J. Theory of ice premelting in porous media. Phys. Rev. E 2010, 81, 031604.

(78) Idziak, S. H.; Safinya, C. R.; Hill, R. S.; Kraiser, K. E.; Ruths, M.; Warriner, H. E.; Steinberg, S.; Liang, K. S.; Israelachvili, J. N. The X-ray surface forces apparatus: structure of a thin smectic liquid crystal film under confinement. Science 1994, 264, 1915-1915.

(79) Idziak, S. H. J.; Safinya, C. R.; Sirota, E. B.; Bruinsma, R. F.; Liang, K. S.; Israelachvili, J. N. Structure of Complex Fluids under Flow and Confinement. ACS Symp. Ser. Am. Chem. Soc. 1994, 578, 288-299.

(80) Idziak, S.; Koltover, I.; Liang, K.; Israelachvili, J.; Safinya, C. Study of flow in a smectic liquid crystal in the X-ray surface forces apparatus. Int. J. Thermophys. 1995, 16, 299-307.

(81) Idziak, S. H.; Koltover, I.; Israelachvili, J. N.; Safinya, C. R. Structure in a confined smectic liquid crystal with competing surface and sample elasticities. Phys. Rev. Lett. 1996, 76, 1477.

(82) Koltover, I.; Idziak, S.; Davidson, P.; Li, Y.; Safinya, C.; Ruths, M.; Steinberg, S.; Israelachvili, J. Alignment of complex fluids under confinement and flow. J. Phys. II 1996, 6, 893-907.

(83) Li, Y.; Golan, Y.; Martin-Herranz, A.; Pelletier, O.; Yasa, M.; Israelachivili, J.; Safinya, C. In situ X-ray diffraction studies of a multilayered membrane fluid under confinement and shear. Int. J. Thermophys. 2001, 22, 1175-1184.

(84) Golan, Y.; Seitz, M.; Luo, C.; Martin-Herranz, A.; Yasa, M.; Li, Y.; Safinya, C. R.; Israelachvili, J. The X-ray surface forces apparatus for simultaneous X-ray diffraction and direct normal and lateral force measurements. Rev. Sci. Instrum. 2002, 73, 2486-2488.
(85) Becker, T. Collapse Dynamics of Confined Liquid Films. Ph.D. Thesis, University of Twente, 2005.

(86) Nygård, K.; Kjellander, R.; Sarman, S.; Chodankar, S.; Perret, E.; Buitenhuis, J.; Van der Veen, J. Anisotropic pair correlations and structure factors of confined hard-sphere fluids: an experimental and theoretical study. Phys. Rev. Lett. 2012, 108, 037802.

(87) Perret, E.; Nygård, K.; Satapathy, D. K.; Balmer, T.; Bunk, O.; Heuberger, M.; van der Veen, J. X-ray reflectivity reveals equilibrium density profile of molecular liquid under nanometre confinement. EPL 2009, 88, 36004.

(88) Perret, E. Structure of Molecular Liquids under Nanometre Confinement. Ph.D. Thesis, ETH Zürich, 2010.

(89) Perret, E.; Nygård, K.; Satapathy, D. K.; Balmer, T. E.; Bunk, O.; Heuberger, M.; van der Veen, J. F. Molecular liquid under nanometre confinement: density profiles underlying oscillatory forces. J. Phys.: Condens. Matter 2010, 22, 235102.

(90) Perret, E.; Nygård, K.; Satapathy, D. K.; Balmer, T. E.; Bunk, O.; Heuberger, M.; van der Veen, J. F. X-ray reflectivity theory for determining the density profile of a liquid under nanometre confinement. J. Synchrotron Radiat. 2010, 17, 465-472.

(91) Seeck, O. H.; Kim, H.; Lee, D. R.; Shu, D.; Kaendler, I. D.; Basu, J. K.; Sinha, S. K. Observation of thickness quantization in liquid films confined to molecular dimension. EPL 2002, 60, 376-382.

(92) Lippmann, M.; Ehnes, A.; Seeck, O. An x-ray setup to investigate the atomic order of confined liquids in slit geometry. Rev. Sci. Instrum. 2014, 85, 015106.

(93) Lippmann, M.; Seeck, O. H.; Ehnes, A.; Nygard, K.; Bertram, F.; Ciobanu, A. Experimental Observation of Crystal-Liquid Coexistence in Slit-Confined Nonpolar Fluids. J. Phys. Chem. Lett. 2019, 10, 16341638.

(94) Tomita, K.; Mizukami, M.; Nakano, S.; Ohta, N.; Yagi, N.; Kurihara, K. X-Ray diffraction and resonance shear measurement of nano-confined ionic liquids. Phys. Chem. Chem. Phys. 2018, 20, 1371413721.

(95) Kuhl, T.; Smith, G.; Majewski, J.; Hamilton, W.; Alcantar, N. Investigating confined complex fluids with neutron reflectivity. Neutron News 2003, 14, 29-31.

(96) Kuhl, T. L.; Smith, G. S.; Israelachvili, J. N.; Majewski, J.; Hamilton, W. Neutron confinement cell for investigating complex fluids. Rev. Sci. Instrum. 2001, 72, 1715-1720.

(97) Hamilton, W.; Smith, G.; Alcantar, N.; Majewski, J.; Toomey, R.; Kuhl, T. Determining the density profile of confined polymer brushes with neutron reflectivity. J. Polym. Sci., Part B: Polym. Phys. 2004, 42, 3290-3301.

(98) de Vos, W. M.; Mears, L. L. E.; Richardson, R. M.; Cosgrove, T.; Dalgliesh, R. M.; Prescott, S. W. Measuring the structure of thin soft matter films under confinement: A surface-force type apparatus for neutron reflection, based on a flexible membrane approach. Rev. Sci. Instrum. 2012, 83, 113903.

(99) Abbott, S. B.; de Vos, W. M.; Mears, L. L. E.; Cattoz, B.; Skoda, M. W. A.; Barker, R.; Richardson, R. M.; Prescott, S. W. Is osmotic pressure relevant in the mechanical confinement of a polymer brush? Macromolecules 2015, 48, 2224-2234.

(100) de Vos, W. M.; Mears, L. L. E.; Richardson, R. M.; Cosgrove, T.; Barker, R.; Prescott, S. W. Nonuniform Hydration and Odd Even Effects in Polyelectrolyte Multilayers under a Confining Pressure. Macromolecules 2013, 46, 1027-1034.

(101) Wei, Z.; Prescott, S. W. Scattering approaches to probing surface layers under confinement. Curr. Opin. Colloid Interface Sci. 2015, $20,253-260$.

(102) Mears, L. L. E. Adsorption and Confinement Effects in Liquid Crystals. Ph.D. Thesis, University of Bristol, 2015.

(103) Wolff, M.; Akgun, B.; Walz, M.; Magerl, A.; Zabel, H. Slip and depletion in a Newtonian liquid. EPL 2008, 82, 36001.

(104) Wolff, M.; Gutfreund, P.; Rühm, A.; Akgun, B.; Zabel, H. Nanoscale discontinuities at the boundary of flowing liquids: a look into structure. J. Phys.: Condens. Matter 2011, 23, 184102. 
(105) Wolff, M.; Kuhns, P.; Liesche, G.; Ankner, J. F.; Browning, J. F.; Gutfreund, P. Combined neutron reflectometry and rheology. J. Appl. Crystallogr. 2013, 46, 1729-1733.

(106) Schwoerer, F.; Trapp, M.; Ballauff, M.; Dahint, R.; Steitz, R. Surface-Active Lipid Linings under Shear Load-A Combined in-Situ Neutron Reflectivity and ATR-FTIR Study. Langmuir 2015, 31, 11539-11548.

(107) Struth, B.; Hyun, K.; Kats, E.; Meins, T.; Walther, M.; Wilhelm, M.; Grübel, G. Observation of new states of liquid crystal 8CB under nonlinear shear conditions as observed via a novel and unique rheology/small-angle $\mathrm{x}$-ray scattering combination. Langmuir 2011, 27, 2880-2887.

(108) Miller, R.; Ferri, J. K.; Javadi, A.; Krägel, J.; Mucic, N.; Wüstneck, R. Rheology of interfacial layers. Colloid Polym. Sci. 2010, 288, 937-950.

(109) Mucic, N.; Javadi, A.; Kovalchuk, N.; Aksenenko, E.; Miller, R. Dynamics of interfacial layers? experimental feasibilities of adsorption kinetics and dilational rheology. Adv. Colloid Interface Sci. 2011, 168, 167-178.

(110) Stamou, D.; Gourdon, D.; Liley, M.; Burnham, N. A.; Kulik, A.; Vogel, H.; Duschl, C. Uniformly flat gold surfaces: imaging the domain structure of organic monolayers using scanning force microscopy. Langmuir 1997, 13, 2425-2428.

(111) Liley, M.; Keller, T. A.; Duschl, C.; Vogel, H. Direct observation of self-assembled monolayers, ion complexation, and protein conformation at the gold/water interface: an ftir spectroscopic approach. Langmuir 1997, 13, 4190-4192.

(112) Song, B.; Walczyk, W.; Schönherr, H. Contact angles of surface nanobubbles on mixed self-assembled monolayers with systematically varied macroscopic wettability by atomic force microscopy. Langmuir 2011, 27, 8223-8232.

(113) Vogel, N.; Zieleniecki, J.; Köper, I. As flat as it gets: ultrasmooth surfaces from template-stripping procedures. Nanoscale 2012, 4, 38203832 .

(114) Huisman, W. J.; Peters, J.; Derks, J.; Ficke, H.; Abernathy, D.; Van Der Veen, J. A new x-ray diffraction method for structural investigations of solid-liquid interfaces. Rev. Sci. Instrum. 1997, 68, $4169-4176$

(115) Reichert, H.; Honkimäki, V.; Snigirev, A.; Engemann, S.; Dosch, $\mathrm{H}$. A new X-ray transmission-reflection scheme for the study of deeply buried interfaces using high-energy microbeams. Phys. B 2003, 336, $46-55$.

(116) Honkimäki, V.; Reichert, H.; Okasinski, J.; Dosch, H. X-ray optics for liquid surface/interface spectrometers. J. Synchrotron Radiat. 2006, 13, 426-431.

(117) Snigirev, A. A.; Filseth, B.; Elleaume, P.; Klocke, T.; Kohn, V.; Lengeler, B.; Snigireva, I.; Souvorov, A.; Tuemmler, J. Refractive lenses for high-energy X-ray focusing. Proc. SPIE 1997, 3151, 164-170.

(118) Snigirev, A.; Snigireva, I.; Vaughan, G.; Wright, J.; Rossat, M.; Bytchkov, A.; Curfs, C. High energy X-ray transfocator based on $\mathrm{Al}$ parabolic refractive lenses for focusing and collimation. J. Phys. Conf. Ser. 2009, 186, 012073.

(119) Hoskin, M. Orientations of Neolithic monuments of Brittany: (2) The early dolmens. J. Hist. Astron. 2007, 38, 487-492.

(120) Mezger, M.; Reichert, H.; Ocko, B. M.; Daillant, J.; Dosch, H. Comment on "How Water Meets a Very Hydrophobic Surface". Phys. Rev. Lett. 2011, 107, 249801.

(121) Timneanu, N. I.; Caleman, C.; Hajdu, J.; van der Spoel, D. Auger electron cascades in water and ice. Chem. Phys. 2004, 299, 277283.

(122) Hubbell, J. H.; Seltzer, S. M. Tables of X-Ray Mass Attenuation Coefficients and Mass Energy-Absorption Coefficients from $1 \mathrm{keV}$ to $20 \mathrm{MeV}$ for Elements $Z=1$ to 92 and 48 Additional Substances of Dosimetric Interest; National Institute of Standards and Technology: Gaithersburg, 1996.

(123) Schöder, S.; Reichert, H.; Schröder, H.; Mezger, M.; Okasinski, J. S.; Honkimäki, V.; Bilgram, J.; Dosch, H. Radiation-Induced Premelting of Ice at Silica Interfaces. Phys. Rev. Lett. 2009, 103, 095502.
(124) Safinya, C.; Sirota, E.; Plano, R. Nematic to smectic-A phase transition under shear flow: a nonequilibrium synchrotron $\mathrm{x}$-ray study. Phys. Rev. Lett. 1991, 66, 1986.

(125) Nakano, S.; Mizukami, M.; Ohta, N.; Yagi, N.; Hatta, I.; Kurihara, K. Structural Change in Smectic Liquid Crystal Nanofilm under Molecular-Scale Confinement Measured by Synchrotron X-ray Diffraction. Jpn. J. Appl. Phys. 2013, 52, 035002.

(126) Nakano, S.; Mizukami, M.; Kurihara, K. Effect of confinement on electric field induced orientation of a nematic liquid crystal. Soft Matter 2014, 10, 2110-2115.

(127) De Jeu, W. H. Basic X-ray Scattering for Soft Matter; Oxford University Press, 2016.

(128) Goodby, J. W.; Collings, P. J.; Kato, T.; Tschierske, C.; Gleeson, H. F.; Raynes, P. Handbook of Liquid Crystals; Wiley-VCH, 2014.

(129) Mukherjee, B.; Peter, C.; Kremer, K. Dual translocation pathways in smectic liquid crystals facilitated by molecular flexibility. Phys. Rev. E 2013, 88, 010502.

(130) Saito, M.; Seto, M.; Kitao, S.; Kobayashi, Y.; Kurokuzu, M.; Yoda, Y.; Yamamoto, J. Small and Large Angle Quasi-Elastic Scattering Experiments by Using Nuclear Resonant Scattering on Typical and Amphiphilic Liquid Crystals. J. Phys. Soc. Jpn. 2012, 81, 023001.

(131) Lis, D.; Backus, E. H. G.; Hunger, J.; Parekh, S. H.; Bonn, M. Liquid flow along a solid surface reversibly alters interfacial chemistry. Science 2014, 344, 1138-1142.

(132) Krämer, G.; Hausen, F.; Bennewitz, R. Dynamic shear force microscopy of confined liquids at a gold electrode. Faraday Discuss. 2017, 199, 299-309.

(133) Schöttl, S.; Horinek, D. Aggregation in Detergent-Free Ternary Mixtures with Microemulsion-Like Properties. Curr. Opin. Colloid Interface Sci. 2016, 22, 8-13.

(134) Mezger, M.; Roth, R.; Schröder, H.; Reichert, P.; Pontoni, D.; Reichert, H. Solid-liquid interfaces of ionic liquid solutions-Interfacial layering and bulk correlations. J. Chem. Phys. 2015, 142, 164707.

(135) Brkljaca, Z.; Klimczak, M.; Milicevic, Z.; Weisser, M.; Taccardi, N.; Wasserscheid, P.; Smith, D. M.; Magerl, A.; Smith, A. S. Complementary Molecular Dynamics and X-ray Reflectivity Study of an Imidazolium-Based Ionic Liquid at a Neutral Sapphire Interface. J. Phys. Chem. Lett. 2015, 6, 549.

(136) Sloutskin, E.; Ocko, B. M.; Tamam, L.; Kuzmenko, I.; Gog, T.; Deutsch, M. Surface layering in ionic liquids: an X-ray reflectivity study. J. Am. Chem. Soc. 2005, 127, 7796-7804.

(137) Haddad, J.; Pontoni, D.; Murphy, B. M.; Festersen, S.; Runge, B.; Magnussen, O. M.; Steinrück, H.-G.; Reichert, H.; Ocko, B. M.; Deutsch, M. Surface structure evolution in a homologous series of ionic liquids. Proc. Natl. Acad. Sci. U. S. A. 2018, 115, E1100.

(138) Kashyap, H. K.; Hettige, J. J.; Annapureddy, H. V.; Margulis, C. J. SAXS anti-peaks reveal the length-scales of dual positive-negative and polar-apolar ordering in room-temperature ionic liquids. Chem. Commun. 2012, 48, 5103-5105.

(139) Coles, S. W.; Smith, A. M.; Fedorov, M. V.; Hausenaef, F.; Perkin, S. Interfacial structure and structural forces in mixtures of ionic liquid with a polar solvent. Faraday Discuss. 2018, 206, 427-442.

(140) Atkin, R.; Warr, G. Structure in Confined Room-Temperature Ionic Liquids. J. Phys. Chem. C 2007, 111, 5162-5168.

(141) Kondrat, S.; Wu, P.; Qiao, R.; Kornyshev, A. A. Accelerating charging dynamics in subnanometre pores. Nat. Mater. 2014, 13, 387393.

(142) Forse, A. C.; Griffin, J. M.; Merlet, C.; Carretero-Gonzalez, J.; Raji, A.-R. O.; Trease, N. M.; Grey, C. P. Direct observation of ion dynamics in supercapacitor electrodes using in situ diffusion NMR spectroscopy. Nat. Energy 2017, 2, 16216. 